Pacific

Journal of

Mathematics

ON PRIMITIVE SUBDIVISIONS OF AN ELEMENTARY TETRAHEDRON

J.-M. Kantor AND K.S. SARKaria 


\title{
ON PRIMITIVE SUBDIVISIONS OF AN ELEMENTARY TETRAHEDRON
}

\author{
J.-M. KANTOR AND K.S. SARKARIA
}

\begin{abstract}
A polytope $\boldsymbol{P}$ of 3 -space, which meets a given lattice $\mathbb{L}$ only in its vertices, is called $\mathbb{L}$-elementary. An $\mathbb{L}$-elementary tetrahedron has volume $\geq(1 / 6)$. $\operatorname{det}(\mathbb{L})$, in case equality holds it is called $\mathbb{L}$-primitive. A result of Knudsen, Mumford and Waterman, tells us that any convex polytope $P$ admits a linear simplicial subdivision into tetrahedra which are primitive with respect to the bigger lattice $(1 / 2)^{t} . \mathbb{L}$, for some $t$ depending on $P$. Improving this, we show that in fact the lattice $(1 / 4) . \mathbb{L}$ always suffices. To this end, we first characterize all $\mathbb{L}$-elementary tetrahedra for which even the intermediate lattice $(1 / 2)$.L suffices.
\end{abstract}

\section{Introduction.}

Following Danilov [Da], a polytope will be called ELEMENTARY with respect to a given lattice $\mathbb{L}$ (or $\mathbb{L}$-elementary, or just elementary) iff it meets the lattice $\mathbb{L}$ only in its vertices. For example, it is easy to see that a parallelepiped with vertices in a lattice $\mathbb{L} \subset \mathbb{R}^{3}$ is elementary with respect to $\mathbb{L}$ if and only if its volume equals $|\operatorname{det} \mathbb{L}|$.

A tetrahedron will be called PRIMITIVE ${ }^{1}$ with respect to a lattice $\mathbb{L}$ (or $\mathbb{L}$-primitive, or just primitive) iff its vertices are in $\mathbb{L}$ and its volume is $\frac{1}{6}|\operatorname{det} \mathbb{L}|$. A primitive tetrahedron is elementary because it is one of the 6 tetrahedra of a volume $|\operatorname{det} \mathbb{L}|$ parallelepiped with vertices in $\mathbb{L}$. The converse is false. One obtains a $\mathbb{Z}^{3}$-elementary tetrahedron ABCD whenever a line segment $\mathrm{AB}$, lying in the plane $z=0$, and having no integral points except $\mathrm{A}$ and $\mathrm{B}$, is joined to an analogous general position line segment CD lying in the "adjacent" parallel plane $z=1$, and such tetrahedra can have volume bigger than any given number.

${ }^{1}$ Usage varies a lot, e.g., primitive is "elementary" in [GKZ], and "basic" and "minimal" have also been used; likewise, elementary is "fundamental" in [Re, Rez], "admissible" in $[\mathbf{W h}]$, "lattice free" in [Ka2], etc. 


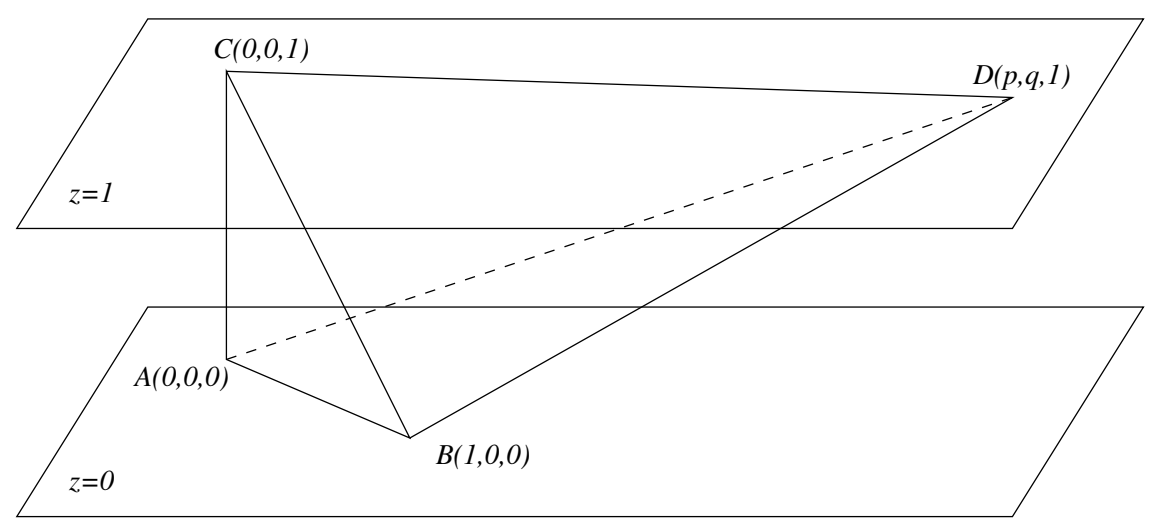

Figure 1.

A theorem of White [Wh] assures us that, upto an ISOMORPHISM, i.e., upto an affine linear transformation which maps $\mathbb{Z}^{3}$ onto itself, the above construction yields all $\mathbb{Z}^{3}$-elementary tetrahedra. It follows (by an argument similar to the proof of Lemma 2 of $\S 2$ ) that, with respect to a suitable origin and basis, we can assume the four vertices to be $A=(0,0,0), B=(1,0,0)$, $C=(0,0,1)$ and $D=(p, q, 1)$, where $0 \leq p \leq q$ with g.c.d. $(p, q)=1$. These standard models of elementary tetrahedra - Figure 1 - will be denoted $\mathbf{T}(p, q)$. Note that the tetrahedron $\mathbf{T}(p, q)$ has volume $q / 6$; so, for all $q \geq 2$, it is non-primitive; the primitive case corresponds to $p=0,1$ and $q=1$, otherwise $1 \leq p<q$.

We note here that in the two dimensional case, the analogues of the above two notions would coincide: An $\mathbb{L}$-elementary triangle must have area $\frac{1}{2}|\operatorname{det} \mathbb{L}|$. For, by adjoining an isomorphic triangle, one can make a parallelogram which is elementary, and so has area $|\operatorname{det} \mathbb{L}|$.

In some arithmetical questions one uses convex cell complexes in $\mathbb{R}^{n}$ having integral vertices. These admit an ELEMENTARY SUBDIVISION with respect to the lattice $\mathbb{Z}^{n}$, i.e., can be linearly subdivided, e.g., by using the method of Hudson $[\mathbf{H u}]$, p. 11, into simplicial complexes having only $\mathbb{Z}^{n}$-elementary simplices. However, for $n \geq 3$, as above examples of nonprimitive elementary tetrahedra already show, not always into primitive simplices. So, to obtain a PRIMITIVE SUBDIVISION, it is necessary to consider, instead of $\mathbb{Z}^{n}$, a bigger lattice, say that of all half-integral points, for which we have the following result:

Theorem 1. A $\mathbf{T}(p, q), q \neq 1$, admits a linear simplicial subdivision, primitive with respect to $\frac{1}{2} \mathbb{Z}^{3}$, iff $p=1$ or $p=q-1$.

It is easily seen that $\mathbf{T}(1, q)$ and $\mathbf{T}(q-1, q)$ are isomorphic, so this theorem tells us that, for each $q \geq 2$, there is, upto isomorphism, just one nonprimitive elementary tetrahedron which can be primitively subdivided by 
using half-integral points. The situation changes dramatically when we are allowed to use quarter-integral points.

Theorem 2. Any $\mathbf{T}(p, q)$ admits a linear simplicial subdivision, primitive with respect to the lattice $\frac{1}{4} \mathbb{Z}^{3}$.

Moreover, our method allows, as one passes in the subdivision process from $\mathbb{Z}^{3}$ to $\frac{1}{2} \mathbb{Z}^{3}$ and then from $\frac{1}{2} \mathbb{Z}^{3}$ to $\frac{1}{4} \mathbb{Z}^{3}$, to subdivide the faces of the tetrahedron in a standard way (each triangular face is cut up into four smaller triangles by joining the mid-points of its three sides to each other). This stronger Theorem $\mathbf{2}^{\prime}$ ensures that the subdivisions of the constituent elementary simplices of a complex fit together, i.e., that one also has the following more general result:

Theorem 3. A convex cell complex with vertices in $\mathbb{Z}^{3}$ always admits a $\frac{1}{4} \mathbb{Z}^{3}$-primitive subdivision.

This improves on a theorem of Knudsen, Mumford and Waterman given in Kempf-Knudsen-Mumford-St.Donat [KKMS] — which asserts the existence of a $\left(\frac{1}{2}\right)^{t} \mathbb{Z}^{3}$-primitive subdivision for some integer $t$ depending on the given cell complex. However, we note that these authors prove their result for any $\mathbb{R}^{n}$, and that they check that their linear subdivisions are projective, ${ }^{2}$ an aspect of the matter which we will ignore in this paper; also (see §3) this weaker result generalizes to polyhedral non-convex cells, while convexity of the cells is needed if one wants to use only quarter-integral points.

Note by J.-M. Kantor. The method of this paper was worked out completely, without any knowledge of previous work, by K.S. Sarkaria. It was only after this paper was written up (as preprint IHES/M/01/23) that I remembered, and passed on to my co-author, some quite incomprehensible drawings which had been given to me by Ziegler, and which apparently had been shown during a talk given by him in 1997 .

Note by K.S. Sarkaria. The problem settled by Theorem 3 was first posed to me in 1995 by J.-M. Kantor; however what eventually sparked this work (done during the time period November 2000 to January 2001) were some stimulating conversations which I had with him much later, during the month of October 2000, when I was visiting Bures-sur-Yvette. I am also grateful to Jean-Michel for checking the arguments of this paper.

Subsequently, I have learnt, by deciphering the aforementioned "incomprehensible drawings", that this work connects with unpublished (actually still to be written out) work of Jeff Lagarias and Günter Ziegler done in

\footnotetext{
${ }^{2}$ We refer the reader to [KKMS] for this definition, as well as for the importance of these results for the theory of toric varieties.
} 
1997, e.g., they had a different method, which apparently also gives a $\mathbb{Z}^{3}$ primitively subdivided $4 \mathbf{T}(p, q)$, however with boundary not subdivided in a standard way, thus it does not yield Theorem 3. I suspect that, if one does not impose any condition on the boundary subdivision, then even $3 \mathbf{T}(p, q)$ is $\mathbb{Z}^{3}$-primitively triangulable. In any case, I have checked this for $q \equiv \pm 1 \bmod p$. All in all, dispensing with the boundary condition should make many tetrahedral problems much easier, a theme I hope to pursue elsewhere.

Outline of the paper. In $\S 2$, we first show that if $\mathbf{T}(p, q)$ has a $\frac{1}{2} \mathbb{Z}^{3}$ primitive subdivision, then, after a preliminary normalization, this subdivision must contain two special edge paths, $X_{1} X_{2} \ldots X_{q-1}$ and $X_{\pi(1)} X_{\pi(2)} \ldots$ $X_{\pi(q-1)}$, which we call "maximal chains". Here, the $X_{i}$ 's denote, in a certain specific order, the $q-1$ points of the lattice $\frac{1}{2} \mathbb{Z}^{3}$ which lie in the interior of the "central parallelogram" (see Figure 2) of $\mathbf{T}(p, q)$, and $\pi$ is a certain permutation of $\{1,2, \ldots, q-1\}$ depending only on $p$ and $q$. So it is necessary that the intersection of the two maximal chains be a common subcomplex. The "only if" part of Theorem 1 follows by checking that this obstruction is nontrivial unless $p=1$ or $q-1$. In $\S 3$ we complete the proof of Theorem 1 by constructing, using maximal chains, a $\frac{1}{2} \mathbb{Z}^{3}$-primitive subdivision of $\mathbf{T}(1, q) \simeq \mathbf{T}(q-1, q)$.

It turns out that the same construction gives a $\frac{1}{2} \mathbb{Z}^{3}$-elementary subdivision of any $\mathbf{T}(p, q)$, if we use instead any two "chains", i.e., edge paths determined by vertex subsequences $X_{i_{1}} X_{i_{2}} \ldots X_{i_{t}}$ and $X_{\pi\left(j_{1}\right)} X_{\pi\left(j_{2}\right)} \ldots X_{\pi\left(j_{s}\right)}$, but again such that their intersection is a common subcomplex. This gives a simple proof of the result of Knudsen, Mumford and Waterman, after which we insert an example of a non-convex polytope with six integral vertices which does not admit a $\mathbb{Z}^{3}$-elementary simplicial subdivision, to show exactly where convexity was used. If an "almost maximal" (see $\S 3$ for the definition) pair of chains is used, each non-primitive $\frac{1}{2} \mathbb{Z}^{3}$-elementary tetrahedron of this subdivision is isomorphic to a $\frac{1}{2} \mathbf{T}(1, t)$, and using Theorem 1 we can subdivide again to obtain a $\frac{1}{4} \mathbb{Z}^{3}$-primitive subdivision. So, the rest of $\S 3$ is devoted to constructing various pairs of almost maximal chains whose intersection is a common subcomplex. First, the special but quite interesting constructions of Proposition 7, and the ensuing Remark 7, which already give numerous instances of $\frac{1}{4} \mathbb{Z}^{3}$-primitive subdivisions, and then the general "quadrant construction" which yields Theorem 2.

Our problem belongs to 3-dimensional affine geometry, and fittingly, the solution we have given below is also pleasingly geometrical; except for the fact that we make an essential use of the theorem of White, and all proofs of this purely geometrical result seem to involve some number theory; also, no satisfactory higher dimensional analogue of this result is known. Before 
starting work we recall below some standard simplicial definitions (for more see e.g., $[\mathbf{H u}])$.

Notations. The convex hull of a finite set of points is called a convex cell, the extreme points being its vertices. If these happen to be affinely independent the cell becomes a simplex, i.e., since we work in 3-space, a point, edge, triangle or tetrahedon. A finite set $K$ of simplices (likewise of cells) with pairwise disjoint interiors, constitutes a complex if the faces of each $\sigma \in K$ are also in $K$. Then we say that $K$ is a linear triangulation of the space $|K|$ obtained by taking the union of these $\sigma$ 's. A simplicial complex $L$ with $|L|=|K|$ is a linear simplicial subdivision of $K$ if each simplex of $L$ is contained in a simplex of $K$. An edge path is a one-dimensional simplicial complex, with edges oriented and totally ordered in such a way that the initial vertex of each coincides with the final vertex of the preceding. The open star $\operatorname{St}_{K}(\sigma)$ of a simplex $\sigma \in K$ consists of all open simplices of $K$ having $\sigma$ as a face, and the link $\operatorname{Lk}_{K}(\sigma)$ of $\sigma$ consists of all simplices disjoint from $\sigma$ which join with $\sigma$ to form simplices of $K$. We sometimes identify a simplex with the complex obtained by adjoining all its faces, and a simplicial complex with the space it triangulates.

\section{Obstruction.}

In this section we analyze linear $\frac{1}{2} \mathbb{Z}^{3}$-primitive subdivisions of $\mathbf{T}(p, q)$ and thereby prove the "only if" part of Theorem 1 . Since this is obviously true for $q \leq 4$, we can assume that $q \geq 5$. The strategy roughly is to reduce the 3-dimensional problem concerning the join $A B * C D$ to a 2-dimensional problem concerning the cartesion product $A B \times C D$.

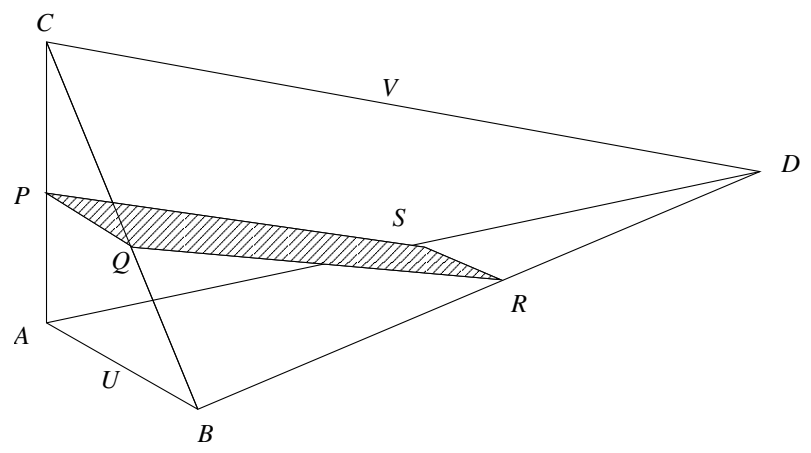

Figure 2.

CENTRAL PARALLELOGRAM. In $\mathbf{T}(p, q)$, the points of the lattice $\frac{1}{2} \mathbb{Z}^{3}$, other than the four vertices $A, B, C, D$, and the six midpoints $P, Q, R$, $S, U, V$ - see Figure 2 - of the edges $A C, B C, B D, A D, A B, C D$ respectively, must all be located in the interior of the parallelogram $P Q R S$, 
i.e., the section of the tetrahedron $\mathbf{T}(p, q)$ by the plane $z=1 / 2$. Note that this parallelogram has area $q / 4$.

THE LATTICE POINTS ON THE PARALLELOGRAM. If we draw lines (Figure 3 shows the case $p=3, q=5$ ) parallel to $P Q$ in the plane $z=\frac{1}{2}$, at equal distances $1 / 2$ from each other, then (lengths equal to $1 / 2$ of) $q-1$ of these lines pass through the interior of our parallelogram, so it follows that there are $q-1$ points of $\frac{1}{2} \mathbb{Z}^{3}$ in the interior of the parallelogram, one on each of these $q-1$ lines (which will sometimes be called HORIZONTALS). We denote these points by $X_{1}, \ldots, X_{q-1}$ in increasing order of their distance from $P Q$. So, $X_{1}$ is the point nearest to $P Q$, while $X_{q-1}$ is the farthest, i.e., the one nearest to $R S$.

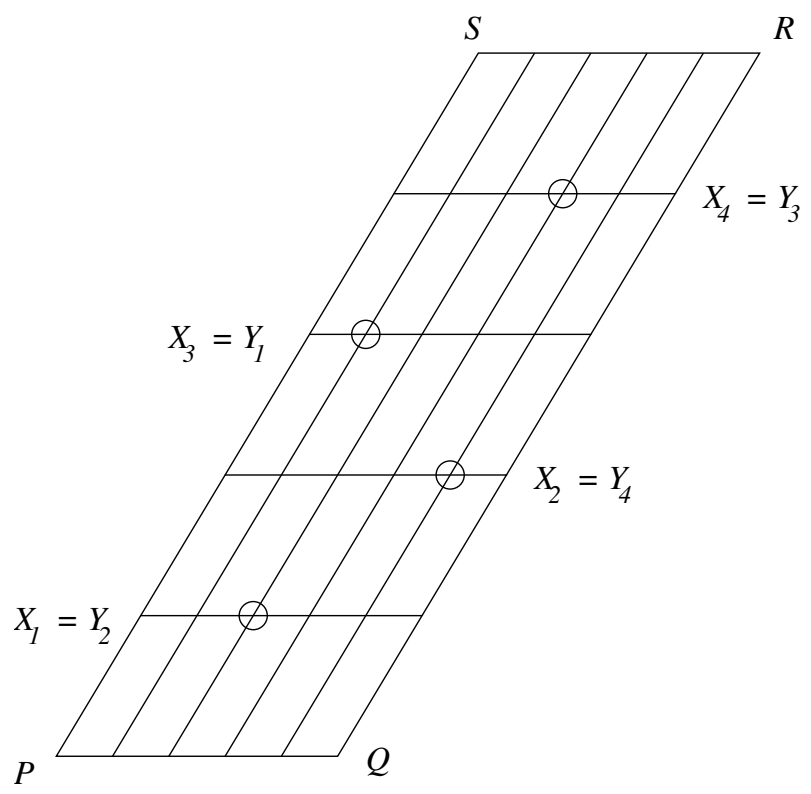

Figure 3.

Likewise, we can subdivide $P Q R S$ into $q$ parallelograms, each of area $1 / 4$, by drawing $q-1$ lines parallel to $S P$, and at equal distances from each other, and precisely one of these points is found on each of these $q-1$ parallels (these will sometimes be called VERTICALS). We will sometimes denote the same set of $q-1$ points (of $\frac{1}{2} \mathbb{Z}^{3}$ in the interior of $P Q R S$ ) by $Y_{1}, \ldots Y_{q-1}$ in increasing order of their distance from $S P$. So, now $Y_{1}$ is the point nearest to $S P$, while $Y_{q-1}$ is the farthest, i.e., the one nearest to $Q R$. We define the PERMUTATION $\pi=\pi(p, q)$ of $\{1,2, \ldots, q-1\}$ by $Y_{j}=X_{\pi(j)}$, and, as mentioned already in $\S 1$, it is this permutation which will play the leading role in the following: 
NORMALIZATION. We begin by showing that we may consider only subdivisions well-behaved with respect to the parallelogram.

Proposition 1. If the elementary tetrahedron $\mathbf{T}=\mathbf{T}(p, q), q \geq 5$, admits a linear simplicial subdivision $\mathbf{K}=\mathbf{K}(p, q)$ which is primitive with respect to $\frac{1}{2} \mathbb{Z}^{3}$, then it also admits another in which the central section occurs as the space $|\mathbf{P}(p, q)|$ of a subcomplex $\mathbf{P}(p, q) \subset \mathbf{K}(p, q)$.

Proof. A simplicial subdivision of our tetrahedron fails to have the parallogram as (the space of) a subcomplex iff it contains an edge having one vertex below it and the other above it. On the boundary of the tetrahedron there are only four possibilities for such an edge, namely $U C, U D, V A, V B$ (e.g., $A C$ is not a possibility because it has $P$ on its interior). In case some or all of these four edges occur in $\mathbf{K}$ we will first get rid of them as follows:

Suppose, e.g., that $U C$ is an edge of $\mathbf{K}$. Then its two incident boundary triangles, which have to be $U P C$ and $U Q C$, must be triangles of $\mathbf{K}$. The two elementary tetrahedra $U P C X_{1}$ and $U Q C X_{1}$ both have volume $1 / 48$, so are primitive with respect to $\frac{1}{2} \mathbb{Z}^{3}$, and all other possible tetrahedra $U P C Z$, $U Q C Z, Z=V, S, R, X_{i}, i \geq 2$, have bigger volumes. So these two tetrahedra must also be in $\mathbf{K}$. We now modify $\mathbf{K}$ by replacing these two tetrahedra, and their common face $U C X_{1}$, by the two primitive tetrahedra $C P Q X_{1}$ and $U P Q X_{1}$, and their common face $P Q X_{1}$. This retriangulation of the open star in $\mathbf{K}$ of the edge $U C$ leaves the triangulations on the other 3 faces of our tetrahedron unaltered. Likewise, if say, $B V \in \mathbf{K}$, then $B V Q Y_{q-1} \in \mathbf{K}$ and $B V R Y_{q-1} \in \mathbf{K}$, and we can get rid of $B V$ by replacing these two tetrahedra and their common face by the primitive tetrahedra $Q R Y_{q-1} B, Q R Y_{q-1} V$ and their common face, etc.

In case $q$ (and so also $p+1$ ) is even, our modified $\mathbf{K}$, which now contains none of the four edges $U C, U D, V A, V B$, has no edge joining a vertex below the parallelogram to one above, and so must contain a subcomplex $\mathbf{P}$ of the required kind. To see this note that $U V$ is not a possible edge of $\mathbf{K}$, because its mid-point $M=\frac{1}{4}(p+1, q, 2) \in \frac{1}{4} \mathbb{Z}^{3}$ - i.e., the BARYCENTER $M$ of $\mathbf{T}(p, q)$, i.e., the intersection of the diagonals of the central section $P Q R S$ - belongs to the lattice $\frac{1}{2} \mathbb{Z}^{3}$ in this case.

In case $q$ is odd, the barycenter $M$ is not contained in $\frac{1}{2} \mathbb{Z}^{3}$, and $U V$ remains a possible edge of $\mathbf{K}$ joining a vertex below the parallelogram to one above. We now describe a procedure for getting rid of $U V$.

First, note that if $U V \in \mathbf{K}$, and $U V I J$ is any tetrahedron of $\mathbf{K}$ incident to this edge, then $I$ and $J$ must be both on the parallelogram. Indeed, neither $I$ nor $J$ can be $C, D, A$ or $B$, for otherwise, one of the 4 already excluded edges $U C, U C, V A, V B$ would be in $\mathbf{K}$. The union $\partial \mathbf{M}$ of all such edges $I J$, i.e., the link of $U V$ in $\mathbf{K}$, bounds a polygonal region $\mathbf{M}$ - namely the union of all the triangles $M I J$ - of the parallelogram, which meets $\frac{1}{2} \mathbb{Z}^{3}$ only in its vertices. We equip $\mathbf{M}$ with any triangulation which is elementary 
(so with triangles of area 1/8) with respect to the half integral lattice of the plane $z=1 / 2$ (see Lemma 1 below). We then modify $\mathbf{K}$ by replacing the tetrahedra $U V I J$, and their common faces, by all the simplices obtained by coning the triangulated $\mathbf{M}$ over $U$ and $V$.

This primitive retriangulation of the open star of $U V$ serves to get rid of the edge $U V$. Since the new $\mathbf{K}$ has no edge joining a vertex below the parallelogram to one above, it will have a subcomplex $\mathbf{P}$ which covers this parallelogram.

Remark 1. We can further modify $\mathbf{K}(p, q)$ so that its restriction to each bounding face is STANDARD (see Figure 4): I.e., that it is cut up into four smaller triangles by joining the mid-points of its sides to each other. (While subdividing simplicial complexes, as in $\S 3$, this finesse is useful, because the subdivisions of two tetrahedra sharing a common face will now match on this common face.) For example, if $P B X_{1} Q$ and $P B X_{1} U$ are in $\mathbf{K}(p, q)$, we retriangulate the open star of their common face $P B X_{1}$ by using $Q U X_{1} P$ and $Q U X_{1} B$ and their common face $Q U X_{1}$.

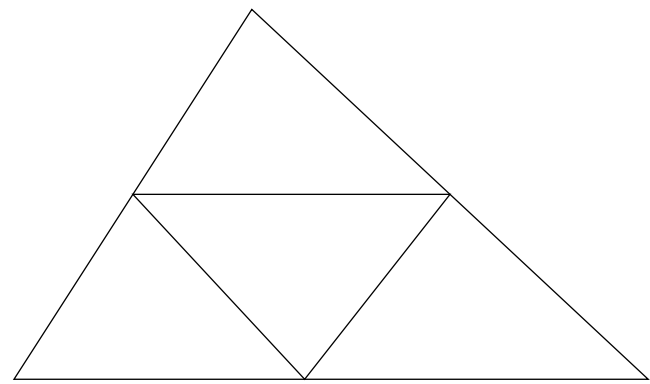

Figure 4.

We insert here a proof of the following which we use in quite a few arguments, e.g., the one just made above. This is an essentially 2-dimensional result, its 3 -dimensional analogue is false (see $\S 3$ ).

Lemma 1. Any (possibly non-convex) $\mathbb{Z}^{2}$-elementary polygonal region $\mathbf{M}$ of $\mathbb{R}^{2}$ can be subdivided into $\mathbb{Z}^{2}$-elementary triangles.

Proof. We use induction on the number $n$ of the vertices $\ldots v_{i-1} v_{i} v_{i+1} \ldots$ of the bounding polygon. If $n=3$ there is nothing to do. If $n \geq 4$, the sum of the $n$ polygonal angles being $(n-2) \pi$, we can choose a vertex $v_{i}$ such that the angle $v_{i-1} v_{i} v_{i+1}$ is less than $\pi$. If the triangle $v_{i-1} v_{i} v_{i+1}$ is elementary, we just add it to any elementary subdivision of the region bounded by the polygon $\ldots v_{i-2} v_{i-1} v_{i+1} \ldots$ having $n-1$ vertices. Otherwise, we take a $u \in \mathbb{Z}^{2}, u \neq v_{i-1}, v_{i}, v_{i+1}$ in this triangle, which is nearest to $v_{i}$. Then $u$ must be a vertex of $\mathbf{M}$, with the interior of the edge $u v_{i}$ contained in the interior of $\mathbf{M}$. Now, using induction, we subdivide the 2 regions into which $\mathbf{M}$ is separated by this edge. 
BOTTOM HALF. We plan next to use the following lemma to derive some conditions on the subcomplex $\mathbf{P}(p, q)$ of Proposition 1 which follow solely by using the primitivity, with respect to $\frac{1}{2} \mathbb{Z}^{3}$, of the tetrahedra of $\mathbf{K}(p, q)$ which cover the portion $z \leq 1 / 2$ of our tetrahedron. This triangulation of the bottom half will be denoted $\mathbf{K}_{X}(p, q)$.

Lemma 2. If $H I J$ is a $\frac{1}{2} \mathbb{Z}^{3}$-elementary triangle on the central parallelogram the $3 \frac{1}{2} \mathbb{Z}^{3}$-elementary tetrahedra AHIJ, UHIJ, BHIJ are all $\frac{1}{2} \mathbb{Z}^{3}$ primitive. Furthermore, if $I J$ is an $\frac{1}{2} \mathbb{Z}^{3}$-elementary edge on the central parallelogram, with $I=\frac{1}{2}\left(i_{1}, i_{2}, 1\right), J=\frac{1}{2}\left(i_{1}, i_{2}, 1\right), j_{2} \geq i_{2}$, then the $\frac{1}{2} \mathbb{Z}^{3}$ elementary tetrahedra AUIJ and UBIJ are isomorphic to each other, and have the standard model $\frac{1}{2} \mathbf{T}\left(j_{1}-i_{1}, j_{2}-i_{2}\right), j_{2} \geq i_{2}$. So these tetrahedra are $\frac{1}{2} \mathbb{Z}^{3}$-primitive if and only if IJ is one of the $q+2$ edges:

$$
P X_{1}, Q X_{1} ; X_{1} X_{2}, X_{2} X_{3}, \ldots, X_{q-2} X_{q-1} ; X_{q-1} S, X_{q-1} R \text {. }
$$

Proof. Because the area of $H I J$ is $1 / 8$, the volume of $A H I J, U H I J$ or $B H I J$ is $1 / 3 \times 1 / 8 \times 1 / 2=1 / 48$, which shows the first part.

Next note (cf. Figure 3) that the first coordinate of $X_{k+1}$ is either the same as that of $X_{k}$, or $\frac{1}{2}$ more. Therefore, $j_{2} \geq i_{2}$ always implies $0 \leq$ $j_{1}-i_{1} \leq j_{2}-i_{2}$. Further, $I J$ has no point of $\frac{1}{2} \mathbb{Z}^{3}$ other than its vertices, iff $j_{1}-i_{1}$ and $j_{2}-i_{2}$ are relatively prime to each other.

For the second part we first give an affine linear transformation which preserves $\frac{1}{2} \mathbb{Z}^{3}$ and maps $A U I J$ onto $U B I J$, viz., the SHEAR which keeps all points of the plane $z=\frac{1}{2}$ fixed, and maps each parallel plane $z=\frac{1}{2}(1-k)$ onto itself by adding $\frac{1}{2} k$ to the first coordinate of its points. Likewise, the $\frac{1}{2} \mathbb{Z}^{3}$-preserving shearing transformation which keeps the plane $z=0$ fixed, and translates $I$ to $P=\frac{1}{2}(0,0,1)$, moves the point $J$ to $\frac{1}{2}\left(j_{1}-i_{1}, j_{2}-i_{2}, 1\right)$, and thus images the tetrahedron $A U I J$ onto the standard model $\frac{1}{2} \mathbf{T}\left(j_{1}-\right.$ $\left.i_{1}, j_{2}-i_{2}\right)$.

It follows that the necessary and sufficient condition for primitivity of these tetrahedra is $j_{2}-i_{2}=1$, which happens if and only if $I J$ is one of the edges listed as $(*)$.

Remark 2. Alternatively, the volume $\frac{1}{6}(A U \times A J)$. AI of $A U I J$, and the volume $\frac{1}{6}(U B \times U J) . U I$ of $U B I J$, are both equal to $1 / 48\left(j_{2}-i_{2}\right)$, being the value of the equal determinants

$$
\frac{1}{48}\left|\begin{array}{ccc}
1 & 0 & 0 \\
j_{1} & j_{2} & 1 \\
i_{1} & i_{2} & 1
\end{array}\right|=\frac{1}{48}\left|\begin{array}{ccc}
1 & 0 & \\
j_{1}-1 & j_{2}-1 & 1 \\
i_{1}-1 & i_{2} & 1
\end{array}\right| .
$$

So the necessary and sufficient condition for primitivity is $j_{2}-i_{2}=1$, which happens iff $I J$ is one of the edges $(*)$. This alone will be used in the present section; however in $\S 3$ we use the fact that these elementary tetrahedra have $" p "=j_{1}-i_{1}$ and " $q "=j_{2}-i_{2}$. 
MAXIMAL CHAIN. The edge path $\mathcal{X}=X_{1} X_{2} \ldots X_{q-2} X_{q-1}$ is called the maximal $X$-chain (likewise $\mathcal{Y}=Y_{1} Y_{2} \ldots Y_{q-2} Y_{q-1}$ will be called the maximal $Y$-chain); note that (*) of Lemma 2 consists of its $q-2 X$-EDGES $X_{i} X_{i+1}$, together with four more: Two each stuck to each end $X_{1}$ and $X_{q-1}$. For small values of $(p, q)$ one can quickly plot the lattice points of the parallelogram on quadrille paper and then draw $\mathcal{X}$ : Figure 5 shows this for the two cases $(p, q)=(4,7)$ and $(3,8)$. We will refer to $P Q X_{1}$ and $X_{q-1} R S$ as $X$-END TRIANGLES, and call the remaining two regions of the parallelogram LEFT $X$-POLYGONAL and RIGHT $X$-POLYGONAL according as it is to the left or right of the chain as we move on it from $X_{1}$ to $X_{q-1}$. Other usages of "left" and "right" will be compatible to the one just made; also, the suffix " $X-$ ", which is used to distinguish these concepts from their obvious " $Y-$ " analogues will often be omitted.

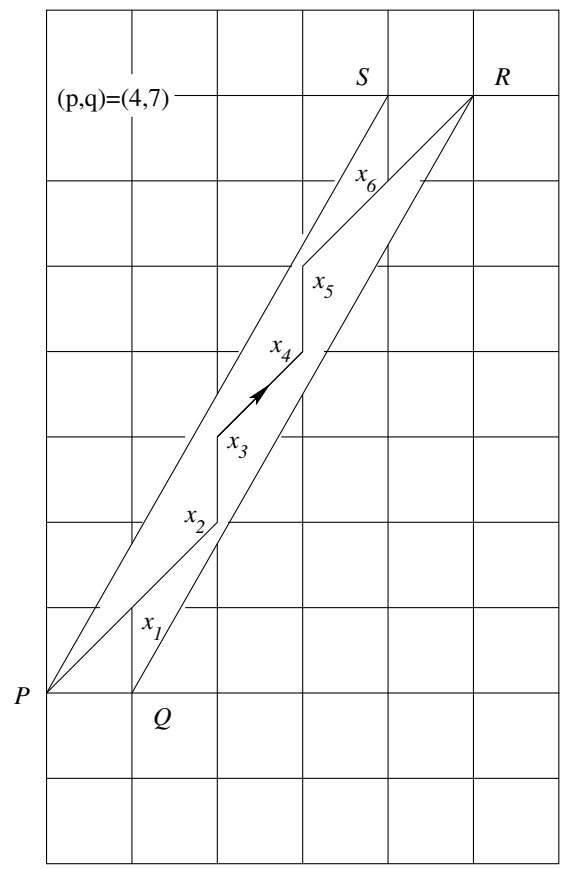

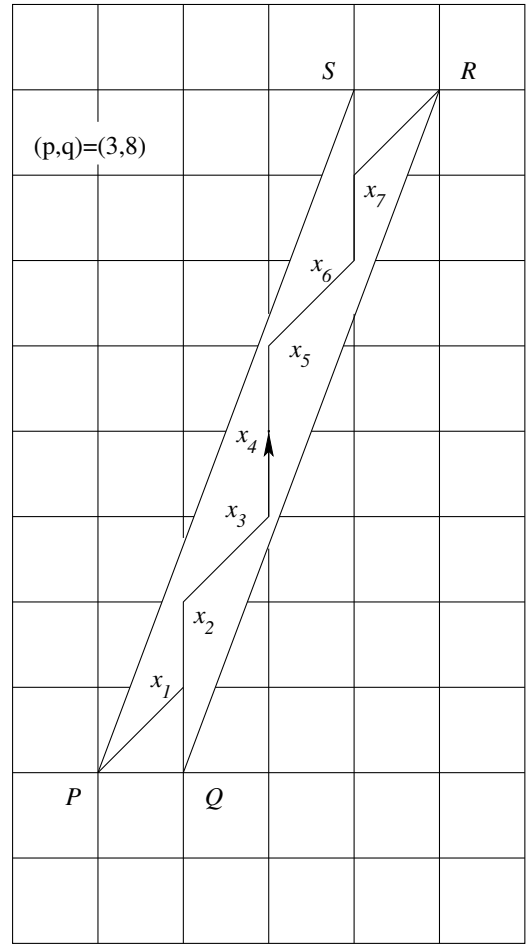

Figure 5 .

WEIGHT. An internal edge $I J$ of the subcomplex $\mathbf{P}(p, q)$ of $\mathbf{K}_{X}(p, q)$, a $\frac{1}{2} \mathbb{Z}^{3}$ primitive simplicial subdivision of the bottom half, will be said to have $X$ weight 0,1 or 2 , depending on whether none, one, or both of the tetrahedra $A U I J$ and $U B I J$ are in $\mathbf{K}_{X}(p, q)$. The following three facts about weight will be important: 
(Wt0) The weight of $I J$ is 0 iff its two incident triangles in $\mathbf{P}(p, q)$ cone over the same vertex from $\{A, U, B\}$ to give tetrahedra of $\mathbf{K}_{X}(p, q)$. For "only if" assume, e.g., that $I J U \in \mathbf{K}_{X}(p, q)$. Since the weight of $I J$ is 0 , the fourth vertices, of the two tetrahedra of $\mathbf{K}_{X}(p, q)$ incident to $I J U$, must be on the parallelogram, i.e., must be the vertices $H_{l}$ and $H_{r}$ - respectively to the left and right of $I J$ - such that $I J H_{l}$, $I J H_{r} \in \mathbf{P}(p, q)$. The converse "if" is obvious.

(Wt1) The weight of $I J$ is 1 , with say $I J A U$ in $\mathbf{K}_{X}(p, q)$ (the statement for $I J U B \in \mathbf{K}_{X}(p, q)$ is analogous), iff (with the same notation as before) $I J H_{l} A$ and $I J H_{r} U$ are in $\mathbf{K}_{X}(p, q)$. For "only if" note that the open tetrahedron $I J A U$ intersects both $I J H_{r} A$ and $I J H_{l} U$. So the fourth vertex of the other tetrahedron incident to $I J A$ has to be $H_{l}$. Again, since weight of $I J$ is 1 , the fourth vertex of the other tetrahedron incident to $I J U$ is also on $\mathbf{P}(p, q)$, and so must be $H_{r}$. For the converse "if" note that, for the fourth vertex of the other tetrahedron incident to $I J A$, there is only one choice, namely $U$.

(Wt2) The weight of $I J$ is 2 iff $A J H_{l} A$ and $A J H_{r} B$ are in $\mathbf{K}_{X}(p, q)$. The verification is similar, so can be omitted.

Proposition 2. For any $\frac{1}{2} \mathbb{Z}^{3}$-primitive simplicial subdivision $\mathbf{K}_{X}(p, q)$ of the bottom half, $z \leq 1 / 2$, of $\mathbf{T}(p, q), q \geq 5$, the subcomplex $\mathbf{P}(p, q)$ covering the parallelogram must contain all the $q+2$ edges $(*)$, and each of the $q-2$ edges $X_{i} X_{i+1}$ must have weight 2 in $\mathbf{P}(p, q)$.

Proof. Since the area of the triangle $P Q Z$ is bigger than $1 / 8$ if $Z=S, R$, or $X_{i}$, the edge $P Q \in \mathbf{P}(p, q)$ is incident to only one elementary triangle of the parallelogram, namely the end triangle $P Q X_{1}$, and so we must have $P Q X_{1} \in \mathbf{P}(p, q)$. Likewise, $X_{q-1} R S \in \mathbf{P}(p, q)$.

The total weight of the edges must be $2 q$. To see this note, since $A, U, B$ are collinear, that any tetrahedron of $\mathbf{K}_{X}(p, q)$ is either of the FIRST KIND, with 3 vertices on the parallelogram, or of the SECOND KIND, with just 2 on the parallelogram, and the total weight is the same as the number of tetrahedra of the second kind. The total volume of all tetrahedra of the first kind is the same as the volume of the pyramid of $P Q R S$ over $B$, i.e., $q / 24$. (Thus the number of tetrahedra of the first kind, i.e., the number of triangles of $\mathbf{P}(p, q)$, is also $q / 24 \div 1 / 48=2 q$.) The remaining volume, i.e., the volume of all tetrahedra of the second kind, is the same as that of the tetrahedron $P A B S$, which is also $q / 24$. This shows that the total number of tetrahedra of the second kind is $q / 24 \div 1 / 48=2 q$.

By Lemma 2 we know all edges which could occur with weight $\geq 1$. So we know (see Figure 5) that, if one of the edges $X_{i} X_{i+1}$ is not in $\mathbf{K}_{X}(p, q)$, or else, even if all these edges are in $\mathbf{K}_{X}(p, q)$, but one of them, $X_{i} X_{i+1}$, has weight 0 , then we can "join" any 2 triangles of $\mathbf{P}(p, q)$, other than the 2 end triangles, by means of a sequence of triangles, such that each shares a 
weight zero edge with the previous one. Using (Wt0), these triangles must all join to the same vertex from $\{A, U, B\}$ and so there are at most 4 edges (of the end triangles) of positive weight. Since $q \geq 5$ the total weight would be less than $2 q$. The entire $X$-chain must thus be in the subcomplex $\mathbf{P}(p, q)$ and all its edges must have positive weight in $\mathbf{K}_{X}(p, q)$.

In case one of its edges $X_{i} X_{i+1}$ is only of weight 1 , say with $A U X_{i} X_{i+1} \in$ $\mathbf{K}_{X}(p, q)$, then by (Wt1), the left triangle of $\mathbf{P}(p, q)$ incident to $X_{i} X_{i+1}$ joins with $\mathrm{A}$, and the right triangle with $\mathrm{U}$, to form tetrahedra of $\mathbf{K}_{X}(p, q)$. Using again the argument of the previous paragraph, it follows that all triangles of the left polygonal region must join with A to give tetrahedra of $\mathbf{K}_{X}(p, q)$, and likewise, all triangles of the right polygonal region must join with $\mathrm{U}$ to form tetrahedra of $\mathbf{K}_{X}(p, q)$. Using the converse part of (Wt1) all edges of the chain must have weight 1 . Since the 2 edges stuck at each end can now contribute at most weight 3 , we see that total weight is at most $(q-2)+2.3=q+4$ which is less than the required $2 q$ because $q \geq 5$. It follows that all edges $X_{i} X_{i+1}$ have weight 2 .

Remark 3. In $\mathbf{K}_{X}(p, q)$ the end triangle $P Q X_{1}$, respectively $X_{q-1} R S$, is incident to one of the tetrahedra from $\left\{A P Q X_{1}, U P Q X_{1}, B P Q X_{1}\right\}$, respectively $\left\{A X_{q-1} R S, U X_{q-1} R S, B X_{q-1} R S\right\}$. In case the boundary faces are subdivided in the standard way (see Remark 1 ) these clearly must be given by the second alternative, i.e., $U P Q X_{1} \in \mathbf{K}_{X}(p, q)$ and $U X_{q-1} R S \in$ $\mathbf{K}_{X}(p, q)$. Also, using (Wt2) and (Wt0), all triangles of the left polygonal region of $\mathbf{P}(p, q)$ must cone over $A$, and all those of the right polygonal region over $B$. Note that now each of the internal edges of an end triangle has weight 1 (if boundary subdivision of that face is not standard one of them will have weight 2 and the other weight 0 ). Thus, with boundary subdivision standard, the triangulation $\mathbf{K}_{X}(p, q)$ is unique, the possible ambiguity being only as to how one chooses to elementarily subdivide the left and right polygonal regions of the parallelogram. [In fact the parallelogram has a unique triangulation with $\mathrm{X}$ a subcomplex, thus there is no ambiguity.]

Remark 4. The above arguments made only a very mild use of the halfintegrality of the vertices $X_{i}$. They show in fact that all the conclusions of Proposition 1 hold even if the $q-1$ vertices $X_{i}$ are any interior points of $P Q R S$, one on each horizontal, provided all the tetrahedra of the subdivision have volume $1 / 48$.

UPPER HALF. The next result gives the analogous conditions, imposed solely by the primitivity, with respect to $\frac{1}{2} \mathbb{Z}^{3}$, of the tetrahedra of $\mathbf{K}(p, q)$ which cover the portion $z \geq 1 / 2$ of our tetrahedron, and can be established by mimicking the proof of Proposition 2. However we give below another proof, in the course of which we will define and use an interesting SYMMETRY $\phi$ between the two halves. 
Proposition 3. For any $\frac{1}{2} \mathbb{Z}^{3}$-primitive simplicial subdivision $\mathbf{K}_{Y}(p, q)$ of the upper half, $z \geq 1 / 2$, of $\mathbf{T}(p, q), q \geq 5$, the subcomplex $\mathbf{P}(p, q)$ covering the parallelogram must contain all the $q+2$ edges:

$$
P Y_{1}, S Y_{1} ; Y_{1} Y_{2}, Y_{2} Y_{3}, \ldots, Y_{q-2} Y_{q-1} ; Y_{q-1} Q, Y_{q-1} R \text {. }
$$

Furthermore, each of the $q-2 Y$-edges $Y_{i} Y_{i+1} \in \mathcal{Y}$ has $Y$-weight 2, i.e., $C V Y_{i} Y_{i+1}$ and $V D Y_{i} Y_{i+1}$ must both be in $\mathbf{K}_{Y}(p, q)$.

For example, for the same values of $(p, q)$ as were used to draw Figure 5, the maximal $Y$-chains $\mathcal{Y}$ are as shown in Figure 6 below (these chains can be made to look less compressed by, say doubling the scale in the $x$-direction).

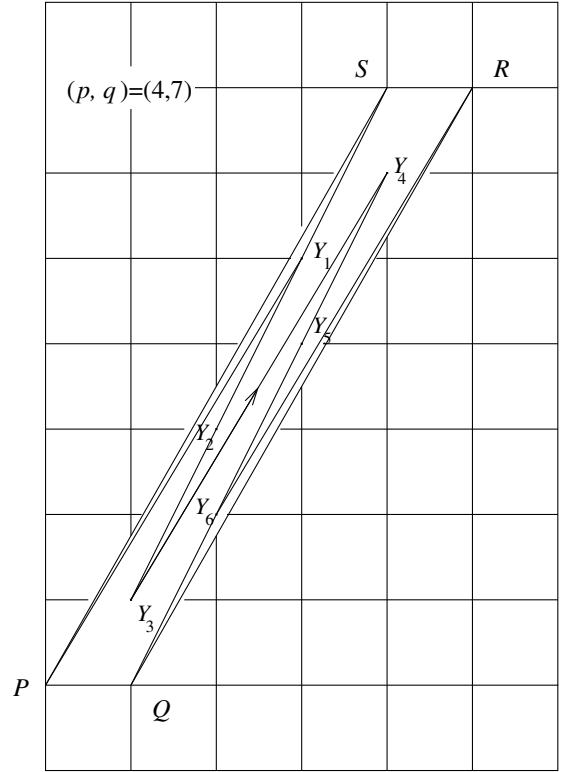

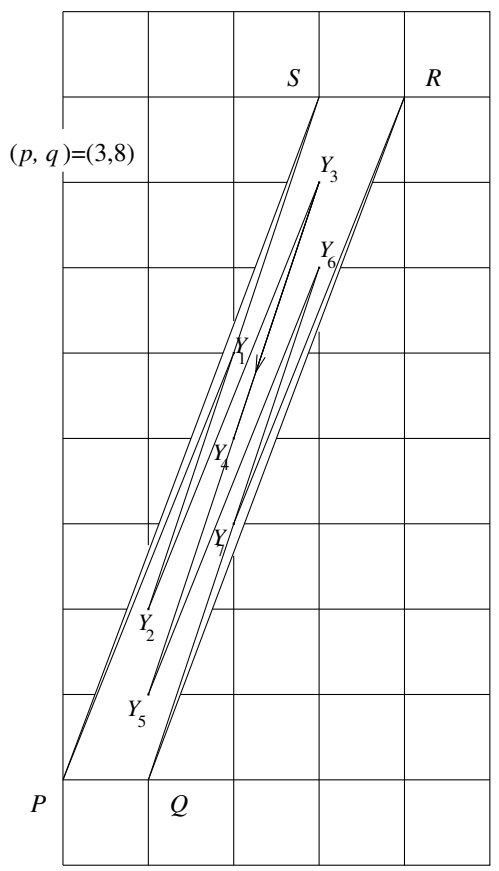

Figure 6.

Proof. The aforementioned $\phi$ will be the linear transformation of 3 -space, having the barycenter $M$ as its fixed point, and mapping $A, B$ and $C$ respectively to $C, D$ and $B$. Since $M A, M B, M C$ and $M D$ add up to the zero vector, it follows that $\phi$ also maps $D$ to $A$. Since $\phi$ preserves the tetrahedron, it must be volume preserving. Moreover, it switches the upper and bottom halves, interchanging the point $V$ with $U$, and "rotates" the parallelogram, mapping $P, Q, R$ and $S$ respectively to $Q, R, S$ and $P$. Its restriction to this parallelogram being area preserving, it follows that $\phi$ must map the straight line through $Y_{i}$ parallel to $S P$ onto the straight line through $X_{i}$ parallel to $P Q$ (however we note that $\phi$ need not map the point $Y_{i}$ to the point $X_{i}$ ).

Applying $\phi$ to the given $\mathbf{K}_{Y}(p, q)$ we obtain a linear subdivision of the bottom half, having $P, Q, R, S, A, U, B$ and $\phi\left(Y_{i}\right), 1 \leq i \leq q-1$, as its 
vertices, with areas of all triangles on the parallelogram $1 / 8$, and volumes of all tetrahedra $1 / 48$. The result now follows by Remark 4 which tells us that all the conclusions of Proposition 1 apply to this combinatorially isomorphic subdivision of the bottom half.

THE OBSTRUCTION. As Figures 5 and 6 suggest, the set theoretic intersection $\mathcal{X} \cap \mathcal{Y}$ is seldom a subcomplex, and so, it is rarely the case that both $\mathcal{X}$ and $\mathcal{Y}$ are subcomplexes of $\mathbf{P}(p, q)$. More precisely, the following concludes the proof of the "only if" part of Theorem 1:

Proposition 4. An $X$-edge of the parallelogram is a $Y$-edge iff $p=1$ or $q-1$ and then all $X$-edges are $Y$-edges. In all other cases there is a pair (X-edge, $Y$-edge) with intersection a non half-integral point.

Proof. All points being now on the parallelogram, their omitted third coordinate will always be $1 / 2$. The segment with end points $I=\frac{1}{2}\left(i_{1}, i_{2}\right)$, $J=\frac{1}{2}\left(j_{1}, j_{2}\right) \in \frac{1}{2} \mathbb{Z}^{3}$ is an $X$-edge iff their second coordinates differ by one, say $j_{2}-i_{2}=1$. Note that the line through $X_{i}$ parallel to SP has equation $q x-p y=k$ where $1 \leq k \leq q-1$ is the integer such that $X_{i}=Y_{k}$. So IJ is also a $Y$-edge iff $\left(q j_{1}-p j_{2}\right)-\left(q i_{1}-p i_{2}\right)=1$ or -1 . Because $1 \leq p<q$, the first case, $q\left(j_{1}-j_{2}\right)=p+1$, occurs iff $q=p+1$ and $j_{1}-i_{1}=1$, and the second case, $q\left(j_{1}-i_{1}\right)=p-1$, happens iff $p=1$ and $j_{1}-i_{1}=0$. In case $p=q-1$ one has $X_{t}=\frac{1}{2}(t, t)=Y_{t}, 1 \leq t<q-1$, and in case $p=1$ one has $X_{t}=\frac{1}{2}(1, t)=Y_{q-t}$ : So in either of these cases the $X$-chain and $Y$-chain coincide and are straight lines. As we will see, in all other cases, both the chains are crooked.

Case $2 p<q$. The slope of the line $S P$ being $q / p$ - see Figure 7 - we will have $X_{1}=\frac{1}{2}(1,1), X_{2}=\frac{1}{2}(1,2), \ldots, X_{t}=\frac{1}{2}(1, t)$, where $t$ denotes the largest integer such that $t p<q$, and then, since $(t+1) p>q$ (as g.c.d. of $p \neq 1$ and $q$ is 1 we cannot have $(t+1) p=q)$ the next $X_{t+1}=\frac{1}{2}(2, t+1)$. So the $X$-chain is crooked with "first bend" at $X_{t}$. Just after this "first bend" of the $X$-chain, there is a non half-integral intersection with a $Y$-edge. To see this note that $X_{1}=(1,1)=Y_{q-p}$ is neither $Y_{1}$ nor $Y_{q-1}$, so the $Y$-chain too is crooked with a bend at this vertex. Also $X_{t+1}=\frac{1}{2}(2, t+1)=Y_{q 2-p(t+1)}$ comes after this vertex in the $Y$-chain because $q 2-p(t+1)>q-p$ is the same as $q>p t$, and $X_{2}=\frac{1}{2}(1,2)=Y_{q-p 2}$ comes before $Y_{q-p-1}$ because $q-p 2<q-p-1$ is the same as $p>1$. So $Y_{q-p-1}$ is trapped in the indicated shaded region and $Y_{q-p-1} Y_{q-p}$ must intersect $X_{t} X_{t+1}$ at an interior point.

Case $2 p>q .^{3}$ The slope of $Q R$ being $q / p$ we now have $X_{1}=\frac{1}{2}(1,1), X_{2}=$ $\frac{1}{2}(2,2), \ldots, X_{t}=\frac{1}{2}(t, t)$, where $t$ is the largest integer such that $t /(t-1)>$ $q / p$. We can have $(t+1) / t=q / p$, i.e., $p(t+1)=q t$, only if $p=t=q-1$,

\footnotetext{
${ }^{3}$ This in fact follows from the case $2 p<q$ because of Lemma 3 below.
} 
which we ruled out; so $(t+1) / t>q / p$ and the next $X_{t+1}=\frac{1}{2}(t, t+1)$. Again, there is an intersection with a $Y$-edge just after this "first bend" of the $X$ chain. This time it is $Y_{q-p+1}$ which is trapped in the shaded region, and so $Y_{q-p} Y_{q-p+1}$ intersects $X_{t} X_{t+1}$. This because $X_{t+1}=\frac{1}{2}(t, t+1)=Y_{q t-p(t+1)}$ comes before $X_{1}=Y_{q-p}$ on the $Y$-chain as $q t-p(t+1)<q-p$ is the same as $q(t-1)<p t$, and $Y_{q-p+1}$ is before $X_{2}$ as $q-p+1<2 q-p 2$ is the same as $p<q-1$.
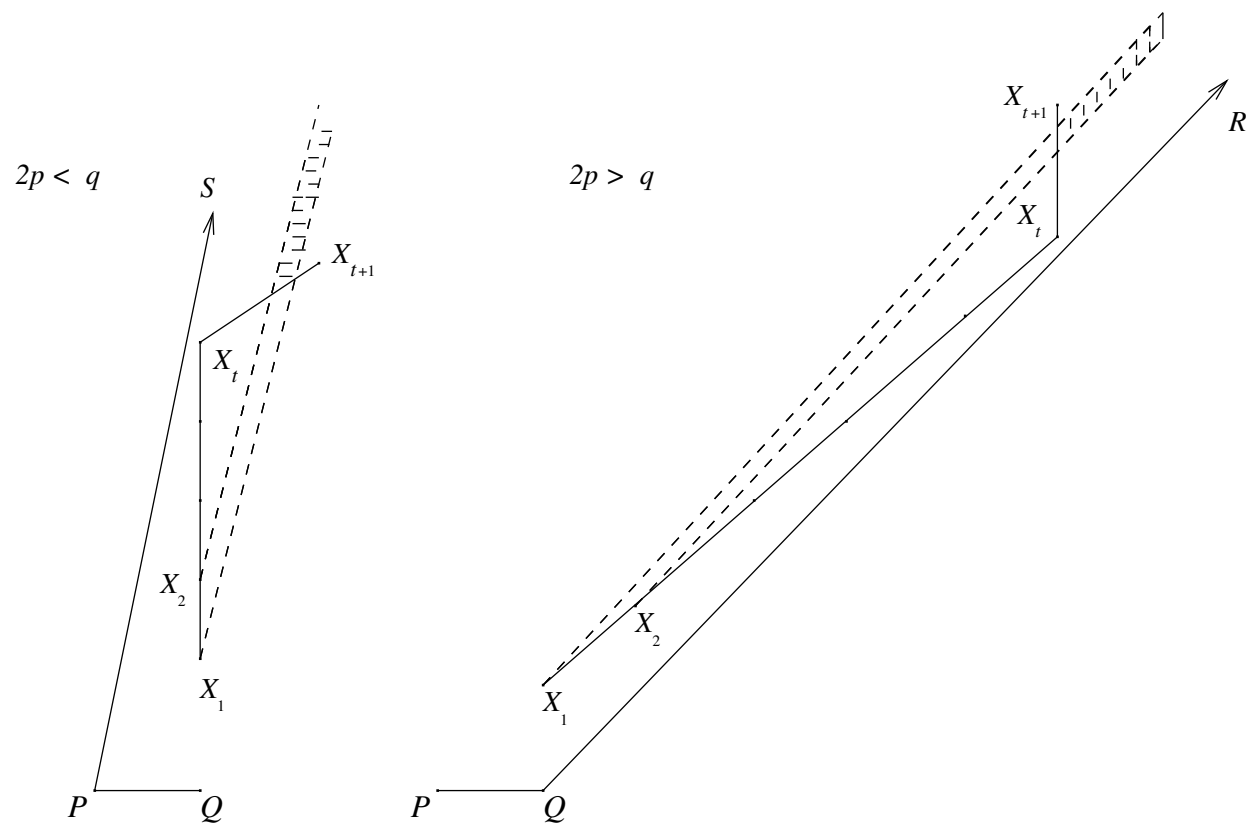

Figure 7.

Remark 5. In the course of the above proof we obtained a more geometric way of stating Theorem 1: An $\mathbb{L}$-elementary tetrahedron admits a $\frac{1}{2} \mathbb{L}$ primitive subdivision iff the points of $\frac{1}{2} \mathbb{L}$ lying in its interior are collinear. Note also that, even though the order 4 volume preserving affine transformation $\phi$ is not lattice preserving, the involution $\phi^{2}$ is (and is in general see proof of Lemma $3, \S 3$ - the sole nonidentity automorphism of $\mathbf{T}(p, q)$ ). It preserves both halves, and, restricted to the central section, reflects it through $M$. The maximal $X$ - and $Y$-chains are thus preserved by reflection through the barycenter $M$, which thus must be on both chains. If $q$ is odd, $M \notin \frac{1}{2} \mathbb{Z}^{3}$, which gives, for this case, another proof of the second part of Proposition 4. 


\section{Construction.}

The analysis of $\S 2$ already indicates - see Remark 3 - what a $\frac{1}{2} \mathbb{Z}^{3}$-primitive subdivision of either half should look like. We now construct such subdivisions. It turns out that the same construction also gives analogous $\frac{1}{2} \mathbb{Z}^{3}$ elementary, but not $\frac{1}{2} \mathbb{Z}^{3}$-primitive subdivisions if we employ non-maximal chains. These are defined as follows:

CHAINS. By an $X$-chain $\mathcal{C}$ we will mean an edge path $X_{i_{1}} X_{i_{2}} \ldots X_{i_{r}}, 1=$ $i_{1}<i_{2}<\cdots<i_{r}=q-1$, such that each of its edges, $X_{i_{t}} X_{i_{t+1}}$, has no halfintegral points in its interior. Its vertices will usually be denoted $\bar{X}_{t}=X_{i_{t}}$, note that the first vertex $\bar{X}_{1}$ is always $X_{1}$, and the last $\bar{X}_{r}$ always $X_{q-1}$. The end triangles and the left/right polygonal regions of $\mathcal{C}$ are defined as for the case of the maximal $X$-chain, and $Y$-chains $\mathcal{D}$ are defined analogously.

SLICING. Using segments $\bar{P}_{t} \bar{Q}_{t}$, parallel to $P Q$, through the vertices $\bar{X}_{t}$ of the given chain $\mathcal{C}$, the bottom half becomes the union of $r-1$ inner slices, i.e., those between the planar sections $\bar{P}_{t} \bar{Q}_{t} A B$ and $\bar{P}_{t+1} \bar{Q}_{t+1} A B, 1 \leq t<r$ and two end slices, between $P Q A B$ and $\bar{P}_{1} \bar{Q}_{1} A B$, and between $\bar{P}_{r} \bar{Q}_{r} A B$ and $S R A B$. As Figure 8 shows, an inner slice subdivides as the union of the tetrahedron $\bar{X}_{t} \bar{X}_{t+1} * A B$ (which subdivides further into two by means of the triangle $\bar{X}_{t} \bar{X}_{t+1} U$ ) and the pyramids of two quadrilaterals over $A$ and $B$. From the first end slice we will carve out the tetrahedron $P Q \bar{X}_{1} U$, leaving us with the pyramid of the quadrilateral $A U \bar{X}_{1} \bar{P}_{1}$ over $P$, and of $U B \bar{Q}_{1} \bar{X}_{1}$ over $Q$, which we will further cut into two parts each by means of the triangles $A P \bar{X}_{1}$ and $B Q \bar{X}_{1}$, respectively. The second end slice is subdivided similarly.
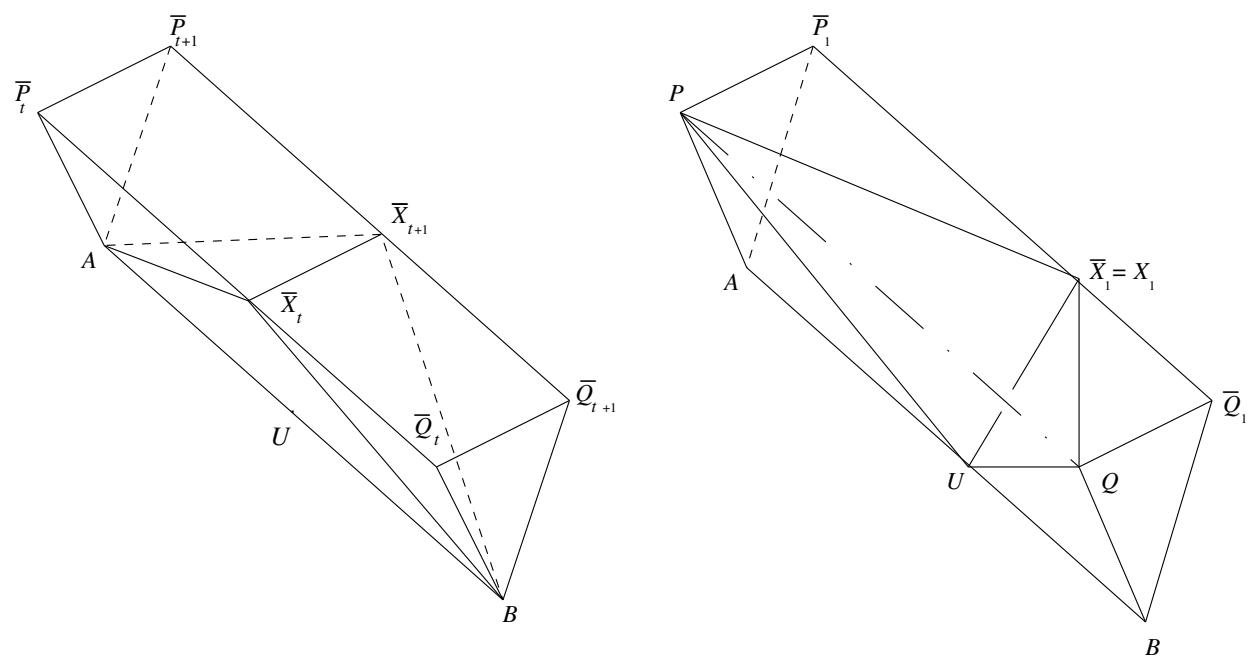

Figure 8. 
Proposition 5. For any $X$-chain $\mathcal{C}$ there is a linear simplicial $\frac{1}{2} \mathbb{Z}^{3}$-elementary subdivision $\mathbf{K}_{\mathcal{C}}(p, q)$ of the bottom half with the following tetrahedra:

(i) Cones over $A$ and $B$, respectively, of triangles of some elementary subdivisions of the left and right polygonal regions of $\mathcal{C}$,

(ii) joins of edges of $\mathcal{C}$ with $A U$ and $U B$, and

(iii) $P A U X_{1}, P Q U X_{1}, Q U B X_{1}$ and $X_{q-1} S A U, X_{q-1} S R U, X_{q-1} R U B$.

Proof. Putting the slices of Figure 8 back together we see that the entire bottom half is the union of (ii) the join of $\mathcal{C}$ with $A B$, (i) $)^{\prime}$ cones over $A$ and $B$ respectively of the left and right polygonal regions of $\mathcal{C}$, and the six tetrahedra (iii). The triangles $\bar{X}_{t} \bar{X}_{t+1} U$ subdivide (ii)' into the tetrahedra (ii). Using Lemma 1 we now elementarily subdivide the left and right polygonal regions, and by coning these over $A$ and $B$, subdivide (i)' into the tetrahedra (i).

Conclusion of proof of Theorem 1. Using Lemma 2 we see that the above tetrahedra (i) and (iii) are primitive, but the tetrahedra of the second kind (ii) are all primitive if and only if $\mathcal{C}=\mathcal{X}$, the maximal $X$-chain. Likewise for the upper half by virtue of the symmetry $\phi$, thus one always has primitive $\frac{1}{2} \mathbb{Z}^{3}$ subdivisions $\mathbf{K}_{\mathcal{X}}(p, q)$ and $\mathbf{K}_{\mathcal{Y}}(p, q)$ for the two halves separately. However, Proposition 4 tells us that, unless $p=1$ or $q-1, \mathcal{X} \cap \mathcal{Y}$ is not a subcomplex, thus $\mathbf{K}_{\mathcal{X}}(p, q)$ and $\mathbf{K}_{\mathcal{Y}}(p, q)$ cannot have the same $\mathbf{P}(p, q)$, and so cannot be put together to obtain a $\frac{1}{2} \mathbb{Z}^{3}$-primitive subdivision of $\mathbf{T}(p, q)$. If $p=1$ or $q-1, \mathcal{X}=\mathcal{Y}$ (except for a change of direction in the $p=1$ case). The subdivisions of the two halves can therefore be chosen with the same $\mathbf{P}(p, q)$, and then fit together to show the remaining "if" part of Theorem 1.

For the sake of completeness we note here that, for the trivial primitive case $q=1$ (now there are no $X_{i}$, so no $\mathcal{C}$ ) we can subdivide the bottom into two tetrahedra, and a pyramid over the central section, as in Figure 9, and cutting the parallelogram into two using either $P R$ or $Q S$, obtain a $\frac{1}{2} \mathbb{Z}^{3}$-subdivision.

$(\mathcal{C}, \mathcal{D})$-SUBDIVISIONS. Given a pair $(X$-chain $\mathcal{C}, Y$-chain $\mathcal{D})$, with $\mathcal{C} \cap \mathcal{D}$ a common subcomplex of both $\mathcal{C}$ and $\mathcal{D}$ (note $\mathcal{C} \cap \mathcal{D} \neq \emptyset$ always) we can, using Lemma 1, find elementary subdivisions $\mathbf{P}(p, q)$ of the parallelogram containing both $\mathcal{C}$ and $\mathcal{D}$ as subcomplexes. Then, using Proposition 5, we can construct an $\frac{1}{2} \mathbb{Z}^{3}$-elementary subdivision $\mathbf{K}_{\mathcal{C}}(p, q) \supset \mathbf{P}(p, q)$ of the bottom half, and analogously, $\mathbf{K}_{\mathcal{D}}(p, q) \supset \mathbf{P}(p, q)$ of the top half. The resultant $\frac{1}{2} \mathbb{Z}^{3}$-elementary subdivision $\mathbf{K}_{\mathcal{C}}(p, q) \cup \mathbf{K}_{\mathcal{D}}(p, q)$ of $\mathbf{T}(p, q)$ will be called a $(\mathcal{C}, \mathcal{D})$-subdivision and denoted by $\mathbf{K}_{\mathcal{C}, \mathcal{D}}(p, q)$. Such pairs $(\mathcal{C}, \mathcal{D})$ always exist, as we show in the course of the following simple proof of the (3-dimensional non-projective) Knudsen-Mumford-Waterman theorem. 


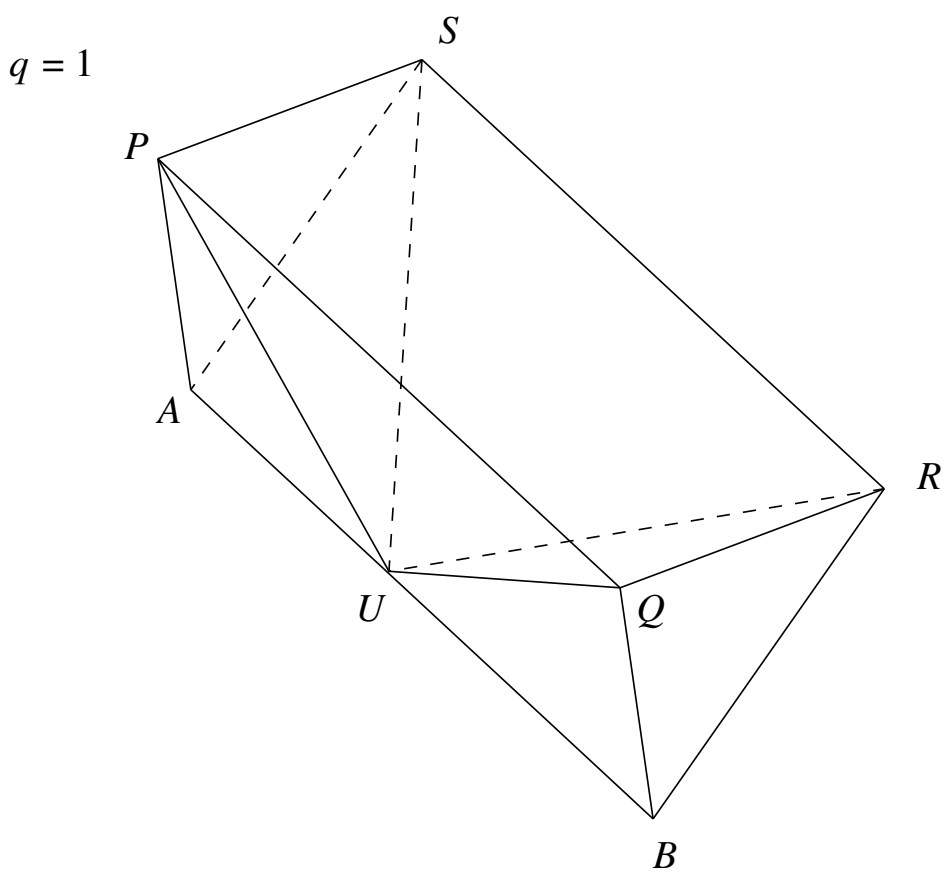

Figure 9.

Proposition 6. Given any finite convex cell complex in $\mathbb{R}^{3}$ with all vertices integral, one can find a $t$ so large that the complex admits a linear simplicial $\left(\frac{1}{2}\right)^{t}-\mathbb{Z}^{3}$ primitive subdivision.

Proof. Because of the convexity of its cells, such a complex can be subdivided simplicially into $\mathbb{Z}^{3}$-elementary simplices. It suffices thus to stipulate an iterative process of elementarily subdividing these simplices (with boundary always to be subdivided in the standard way) which will eventually give, for some $t$ depending on $p$ and $q$, a $\left(\frac{1}{2}\right)^{t}-\mathbb{Z}^{3}$ primitive subdivision (simplices already primitive will always be further subdivided as in Figure 9).

We assume $X_{1}, Y_{q-1}, X_{q-1}, Y_{1}$ are distinct, for otherwise, $p=1$ or $q-1$ and we can attain primitivity in just one step. We join these four points in cyclic order, and then further subdivide this "rhombus" - see Figure 10 - by half-integral points, if any, which lie on the interiors of its edges. The subdivided edge path $X_{1} Y_{q-1} X_{q-1}$ will be our $\mathcal{C}$, and the subdivided edge path $Y_{1} X_{1} Y_{q-1}$ our $\mathcal{D}$, so $\mathcal{C} \cap \mathcal{D}$, i.e., the subdivided edge $X_{1} Y_{q-1}$, is a subcomplex of both. Using Lemma 1 we choose elementary subdivisions of the components of the complement of the "rhombus" and extend this to a subdivision $\mathbf{K}_{\mathcal{C}}(p, q) \cup \mathbf{K}_{\mathcal{D}}(p, q)$. 


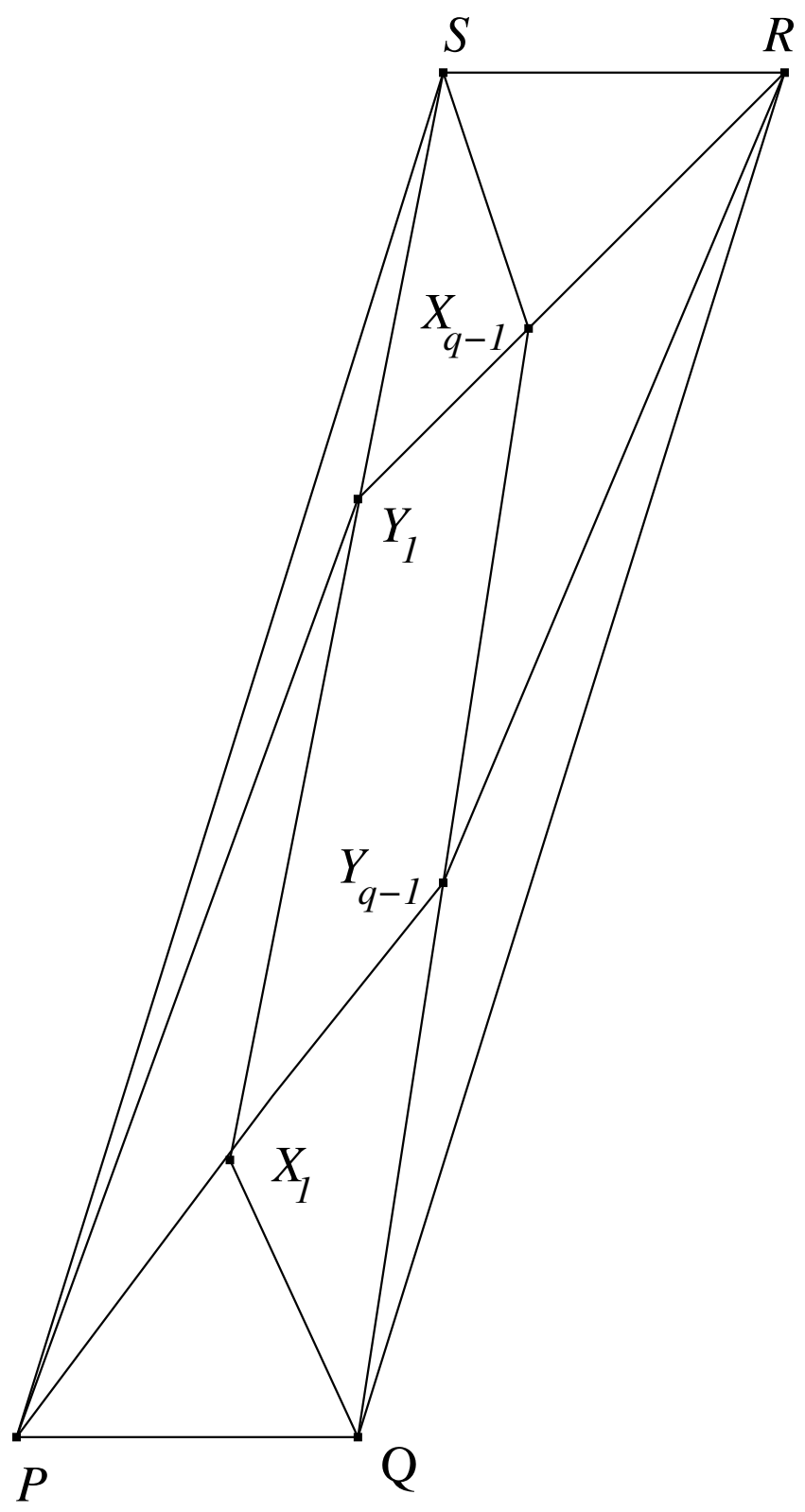

Figure 10.

As $X_{1}$ and $X_{q-1}$, respectively $Y_{1}$ and $Y_{q-1}$, lie on the first and $(q-1)$ th parallels to $P Q$, respectively $S P$, using Lemma 2 and the symmetry $\phi$ we see that the new " $q$ ", of any tetrahedron of this subdivision, is less than $q-2$. So all tetrahedra eventually become primitive. 
We show next that, without convexity, the first step of the last proof i.e., the 3 -dimensional analogue of Lemma 1 - is false.

A NON-CONVEX 3-CELL. There is a region $\Omega$ of $\mathbb{R}^{3}$, whose boundary is uniquely triangulable as a linearly embedded 6-vertex simplicial 2-sphere, which meets $\mathbb{Z}^{3}$ only in these six vertices, and which cannot be simplicially subdivided into elementary tetrahedra.

Two of the eight boundary triangles, $a b c$ and $A B C$, of this non-convex 3 -cell, will lie, respectively, on the horizontal planes $z=0$ and $z=1$, while the remaining six, $\{a b B, a A B\},\{b c C, b B C\}$ and $\{c a A, c C A\}$, will form three sloping quadrilaterals, $a b A B, b c B C$ and $c a C A$, all flexed inwards along the diagonals $a B, b C$ and $c A$. In other words, these three diagonals are on the boundary of $\Omega$, but the remaining three diagonals, $A b, B c$ and $C a$, are all outside $\Omega$. This rules out an elementary simplicial subdivision, because no tetrahedron of such a subdivision could be incident to the face $A B C$ of $\Omega$.

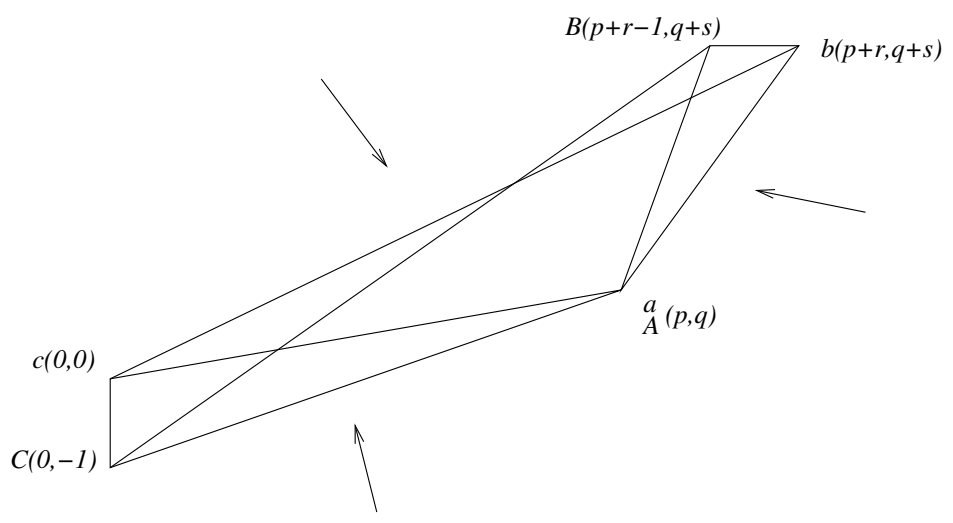

Figure 11.

To arrange this, let the $(x, y)$ projections of the vertices be as in Figure 11, which thus is $\Omega$ viewed from the top. More precisely, let $c=(0,0,0)$, $C=(0,-1,1), a=(p, q, 0), A=(p, q, 1), b=(p+r, q+s, 0), B=(p+$ $r-1, q+s, 1)$, for any four positive integers $p, q, r, s$, satisfying $\left|\begin{array}{ll}p & q \\ r & s\end{array}\right|=1$ and $r=q+1$ (e.g., $p=7, q=4, r=5, s=3$ ). The primitivity of $A B C$ follows from $\left|\begin{array}{cc}p & q+1 \\ r-1 & s\end{array}\right|=\left|\begin{array}{cc}p & r \\ q & s\end{array}\right|=1$ and these equations also ensure that ab slopes more than $c a$, and $A B$ more than $C A$. That the quadrilaterals are flexed inwards on the asserted diagonals follows by viewing them from outside along the indicated arrows.

Note that the above example has the least number of vertices. A simplicial 4 -vertex 2 -sphere is the boundary of a tetrahedron, and a 5 -vertex simplicial 2 -sphere either the boundary of 2 tetrahedra on a common triangle, or 3 tetrahedra around a common edge. 
Remark 6. Nevertheless, we emphasize that Proposition 6 itself is true even if the cells of the complex are not convex. To see this note that all planes, which pass through three affinely independent vertices of the complex, give us a canonical subdivision into smaller convex cells with vertices in a bigger lattice $\frac{1}{l} \mathbb{Z}^{3}$. We choose a simplicial subdivision of this canonical subdivision with vertices in the same lattice $\frac{1}{l} \mathbb{Z}^{3}$. If $l$ contains primes other than 2 , we now move the new vertices slightly to get a combinatorially isomorphic linear simplicial subdivision with vertices in some $\left(\frac{1}{2}\right)^{t}-\mathbb{Z}^{3}$, and then proceed to a primitive subdivision just as before.

To choose more efficient chain pairs $(\mathcal{C}, \mathcal{D})$, we need some more facts regarding the disposition of the $q-1$ points of the lattice $\frac{1}{2} \mathbb{Z}^{3}$ within the central parallelogram.

LATITUDES AND LONGITUDES. The lattice point $X_{2}$ lies either on $Q X_{1}$ produced (this happens iff $q / p>2$ ) or on $P X_{1}$ produced; accordingly we will refer to the straight portions of the maximal $X$-chain parallel to $Q X_{1}$ or $P X_{1}$ as latitudes. Likewise, $Y_{2}$ lies on $P Y_{1}$ produced or on $S Y_{1}$ produced, and accordingly, straight portions of the maximal $Y$-chain parallel to $P Y_{1}$ or $S Y_{1}$ will be called longitudes. Their slopes can be easily computed by using the fact that (besides $z=\frac{1}{2}$ ) the coordinates of the end points are $X_{1}=\frac{1}{2}(1,1), X_{q-1}=\frac{1}{2}(p, q-1), Y_{1}=\frac{1}{2}(r, s)$ and $Y_{q-1}=\frac{1}{2}(p+1-r, q-s)$, where $\left|\begin{array}{ll}r & s \\ p & q\end{array}\right|=1$ (because $P Y_{1} S$ and $R Y_{q-1} S$ are congruent elementary triangles). Note that $1 \leq r<p$ represents the element $q^{-1}$ of $(\mathbb{Z} / p)^{\times}$, and $1 \leq s<q$ is given by $s=-p^{-1} \in(\mathbb{Z} / q)^{\times}$.

This is the number $s$ of Lemma 3 below, a known ${ }^{4}$ classification of the standard models. Then Lemma 4 gives a geometrical reformulation of Lemma 2 and its $Y$-analogue in terms of (latitudes, horizontals) and (longitudes, verticals). Here, lat $(I J)=0$ if the latitudes through $I$ and $J$ coincide, and otherwise lat $(I J)$ is one more than the number of intervening latitudes of the other three nonnegative numbers hor $(I J)$, long $(I J)$ and $\operatorname{ver}(I J)$ are defined analogously.

Lemma 3. $\mathbf{T}(p, q)$ is isomorphic to $\mathbf{T}\left(p^{\prime}, q^{\prime}\right)$ iff $q^{\prime}=q$ and $p^{\prime}$ is one of the four numbers $\{p, q-p, s, q-s\}$.

Lemma 4. If IJ is an $\frac{1}{2} \mathbb{Z}^{3}$-elementary edge of the parallelogram then AUIJ and $U B I J$ are isomorphic to $\frac{1}{2} \mathbf{T}(\operatorname{lat}(I J)$, hor $(I J))$, while $C V I J$ and $V D I J$ are isomorphic to $\frac{1}{2} \mathbf{T}($ long $(I J)$, ver $(I J))$.

Proof. For the "if" part of Lemma 3 note that the affine transformation $(x, y, z) \longrightarrow(-x+y-z+1, y, z)$ maps $\mathbb{Z}^{3}$ onto $\mathbb{Z}^{3}$ and $\mathbf{T}(p, q)$ onto $\mathbf{T}(q-p, q)$.

\footnotetext{
${ }^{4}$ Lemma 3 is a particular case of Theorem 5.6 of Reznick [Rez]; in [Ka], Proposition 8, two of the 4 possibilities were overlooked.
} 
Again, if we use the unimodular matrix $\left[\begin{array}{cc}c-s & -p+r \\ q & -p\end{array}\right]$ to transform each plane $z=$ constant, and then reflect in the central plane $z=\frac{1}{2}$, we get the $\mathbb{Z}^{3}$-preserving affine transformation $\Psi(x, y, z)=(x(q-s)+y(-p+r), x q-$ $y p, 1-z)$, which maps $\mathbf{T}(p, q)$ onto $\mathbf{T}(q-s, q)$.

For the converse note that an affine transformation $\Psi$ will map $\mathbf{T}(p, q)$ onto $\mathbf{T}\left(p^{\prime}, q^{\prime}\right)$ iff $(\Psi(A), \Psi(B), \Psi(C), \Psi(D))$ is one of the $4 !=24$ permutations of the vertices $\left(A^{\prime}, B^{\prime}, C^{\prime}, D^{\prime}\right)$ of the second tetrahedra. Here we use primes to denote corresponding points of second tetrahedron, so $A^{\prime}=A$, $B^{\prime}=B, C^{\prime}=C$ and $D^{\prime}=\left(p^{\prime}, q^{\prime}, 1\right)$. we have $q^{\prime}=q$ because the two tetrahedra have the same volume; so $\Psi$ is volume preserving, and $\Psi\left(\mathbb{Z}^{3}\right)=\mathbb{Z}^{3}$ iff $(0,1,0)=(p / q+1) A-(p / q) B-(1 / q) C+(1 / q) D$ is mapped to an integral point $\Psi(0,1,0)=(p / q+1) \Psi(A)-(p / q) \Psi(B)-(1 / q) \Psi(C)+(1 / q) \Psi(D)$. Checking this for each of the 24 cases (cf. Reznick [Rez]) one sees that such a $\Psi$ exists only if $p^{\prime}=p, q-p, s$ or $q-s$.

For the isomorphism $\Psi: \mathbf{T}(p, q) \simeq \mathbf{T}(q-s, q)$ defined in the first paragraph $(\Psi(A), \Psi(B), \Psi(C), \Psi(D))=\left(C^{\prime}, D^{\prime}, A^{\prime}, B^{\prime}\right)$ so $\Psi$ maps $(P, Q, R, S$, $U, V)$ in order onto $\left(P^{\prime}, S^{\prime}, R^{\prime}, Q^{\prime}, V^{\prime}, U^{\prime}\right)$. From this it follows that it maps the verticals, resp. longitudes, of $\mathbf{T}(p, q)$ onto the horizontals, resp. latitudes, of $\mathbf{T}(q-s, q)$, with the $q-1$ points $Y_{k}$ of the central section of $\mathbf{T}(p, q)$ mapping in order on the $q-1$ points $X_{k}^{\prime}$ of $\mathbf{T}(q-s, q)$.

This last observation shows that it is enough to prove only the first part of Lemma 4. For this note that, by Lemma 2, both $A U I J$ and $U B I J$ are isomorphic to $\frac{1}{2} \mathbf{T}\left(j_{1}-i_{1}, j_{2}-i_{2}\right)$, but hor $(I J)=j_{2}-i_{2}$, and lat $(I J)$ is either the same as $j_{1}-i_{1}$ (this when $q>2 p$ for then the latitudes have equations $x=$ constant) or equal to $\left(j_{2}-i_{2}\right)-\left(j_{1}-i_{1}\right)$ (this when $q<p$ and the latitudes have equations $y-x=$ constant $)$.

For somewhat higher values of $(p, q)$, than those used in Figures 5 and 6 , it is helpful to plot the above four kinds of lines using the following device, because it avoids the congestion of longitudes and verticals, and gives a more balanced picture.

SQUARING. Our problem belongs to three dimensional affine geometry, i.e., all that we are saying about $\mathbf{T}$ and $\mathbb{Z}^{3}$ applies equally well to $\mu(\mathbf{T})$ and $\mu\left(\mathbb{Z}^{3}\right)$, where $\mu$ is any affine transformation of 3 -space. For instance, by applying the linear $\mu: \mathbb{R}^{3} \longrightarrow \mathbb{R}^{3}$ having matrix

$$
\left[\begin{array}{ccc}
q & -p & 0 \\
0 & 1 & 0 \\
0 & 0 & 1
\end{array}\right],
$$

we obtain a $\mu \mathbf{T}$ having edges $A B$ and $C D$ of the same length, and its section $z=\frac{1}{2}$ is a square, the new or "squared" $x$ - and $y$-coordinates of the various points being $\frac{1}{2}$ times the numbers indicated in Figure 12 . 


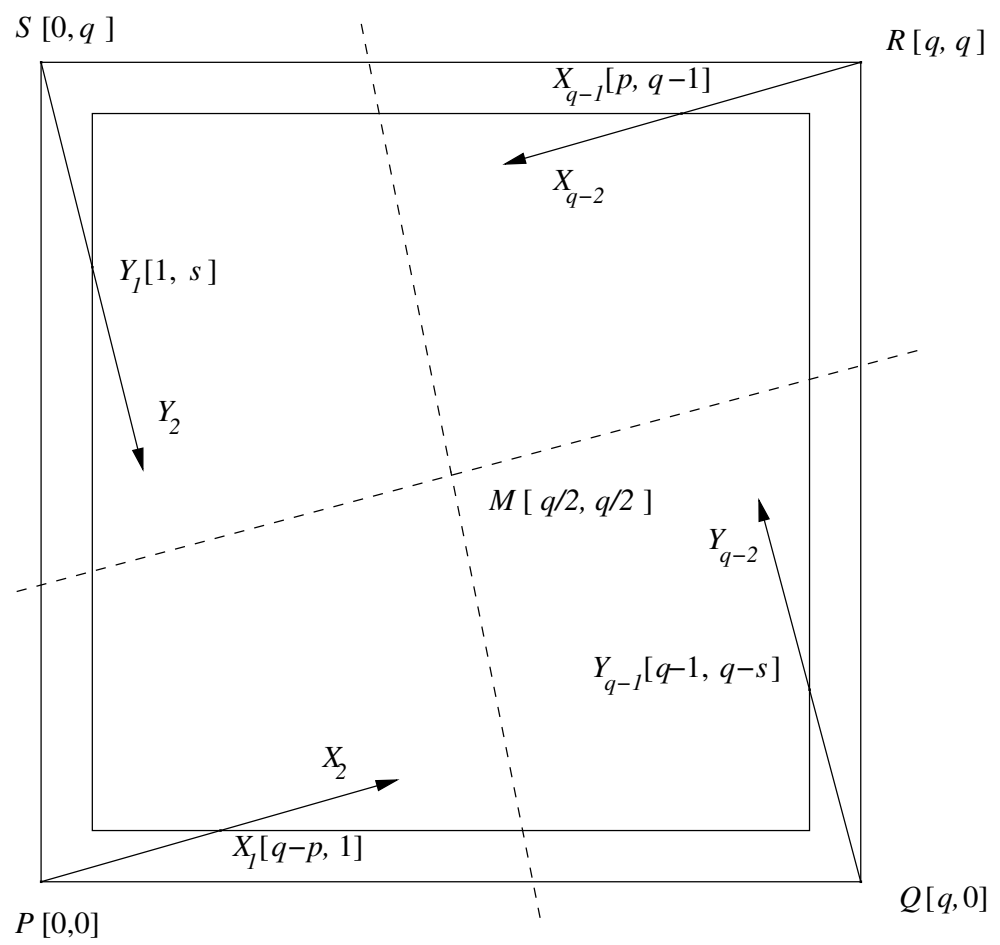

Figure 12.

Note that the $q-1$ equally spaced verticals, being parallel to $S P$, are now in fact "vertical", and the spacing between them is the same as between the $q-1$ horizontals. There is one and only one point of the lattice $\mu\left(\mathbb{Z}^{3}\right)$ on each horizontal and vertical, and these points can be found by starting from any end point and making some obvious MOVES. For example, we start from $X_{1}$ in the direction $P X_{1}$ - because $P X_{1}<Q X_{1}$ in Figure 12, in case $P X_{1}>Q X_{1}$ start in the direction $Q X_{1}$ - till we meet the second horizontal in $X_{2}$, and so on, as long as we stay in the square. Having thus obtained the first latitude $X_{1} X_{2} \ldots X_{i}$, we then make one move from $X_{i}$ parallel to $Q X_{1}$ to find $X_{i+1}$ on the next horizontal, after which we again make moves parallel to $P X_{1}$ and obtain the second latitude, etc. In some figures below, we will only draw the smaller square passing through the four end points. The "crossword" symmetry of these figures reflects that $A \leftrightarrow B, C \leftrightarrow D$ is an automorphism of $\mathbf{T}(p, q)$, and the fact that the end points $X_{q-1}, X_{1}, Y_{1}, Y_{q-1}$ have, respectively, squared coordinates $(p, q-1),(q-p, 1),(1, s),(q-1, q-s)$ is related to Lemma 3: The symmetries of the square $P Q R S$ induce the four isomorphisms $\mathbf{T}(p, q) \simeq \mathbf{T}\left(p^{\prime}, q\right)$. Note that if $p=s$ or $q-s$, then one has only two numbers, and so only two isomorphic tetrahedra; but nevertheless, Lemma 3 shows that one may always assume $p<q / 2$, as we will do in the next proposition. 
ALMOST MAXIMAL CHAINS. An $X$-chain $\mathcal{C}=\bar{X}_{1} \bar{X}_{2} \ldots \bar{X}_{r}$ is called almost maximal if its edges $\bar{X}_{i} \bar{X}_{i+1}$ join vertices on the same or adjacent latitudes, i.e., iff lat $\left(\bar{X}_{i} \bar{X}_{i+1}\right)=0$ or 1 . Thus, besides $\mathcal{X}$, there are many other almost maximal chains; for any such $\mathcal{C}$, Lemma 4 tells us that the $\frac{1}{2} \mathbb{Z}^{3}$-elementary tetrahedra $\bar{X}_{i} \bar{X}_{i+1} * A U$ and $\bar{X}_{i} \bar{X}_{i+1} * U B$ are either $\frac{1}{2} \mathbb{Z}^{3}$ primitive or $\frac{1}{2} \mathbb{Z}^{3}$-ALMOST PRIMITIVE, i.e., isomorphic to $\frac{1}{2} \mathbf{T}(1, t)$ for some $t$. Almost maximal $Y$-chains are defined analogously. So, if $(\mathcal{C}, \mathcal{D})$ is a pair of almost maximal chains, with $\mathcal{C} \cap \mathcal{D}$ a subcomplex, then, by using the "if" part of Theorem 1 to further subdivide the $\frac{1}{2} \mathbb{Z}^{3}$-elementary subdivision $\mathbf{K}_{\mathcal{C}, \mathcal{D}}(p, q)$, we obtain a $\frac{1}{4} \mathbb{Z}^{3}$-primitive subdivision of $\mathbf{T}(p, q)$. As a warm-up for the proof of Theorem 2, we first show that often one of these chains $(\mathcal{C}, \mathcal{D})$ can be chosen arbitrarily.

Proposition 7. The two $X$-chains, which run from $X_{1}$ to $X_{q-1}$ on the boundary of the convex hull, of the $q-1$ points of $\frac{1}{2} \mathbb{Z}^{3}$ lying in the interior of the section $z=\frac{1}{2}$ of $\mathbf{T}(p, q), p<q / 2$, are almost maximal if and only if $p$ divides $q-1$ or $q+1$.

For any such $(p, q)$, we obtain a $\frac{1}{4} \mathbb{Z}^{3}$-primitive subdivision of $\mathbf{T}(p, q)$ by subdividing $\mathbf{K}_{\mathcal{C}, \mathcal{D}}(p, q)$, where $\mathcal{C}$ is one of these two chains, and $\mathcal{D}$ is any almost maximal $Y$-chain. This follows because, $\mathcal{C}$ being on their convex hull, no edge path $\mathcal{D}$, having as vertices some or all of the $q-1$ lattice points can cross it. Thus $\mathcal{C} \cap \mathcal{D}$ is a subcomplex for any $\mathcal{D}$. If $\mathcal{D}$ is maximal, the top half of $\mathbf{K}_{\mathcal{C}, \mathcal{D}}(p, q)$ is already $\frac{1}{2} \mathbb{Z}^{3}$-primitive, and the bigger lattice $\frac{1}{4} \mathbb{Z}^{3}$ is used only to make the bottom primitive.

Proof. We assert that, for $q / p>2$, the maximal $X$-chain has $p$ latitudes, the first having $t=[q / p]$ vertices, and all others having either $t$ or else $t+1$ vertices. This centrally symmetric partition into $p$ parts of size $t$ or $t+1$ will be called the $X$-PARTITION of $q-1$.

To see this note that in this case the latitudes are configured as for the $(3,8)$ picturized in Figure 5, i.e., they lie on $p$ equally spaced "verticals" (not to be confused with verticals (!) which are parallel to $P S$ ). The first of these "verticals" passes through $Q$, and the last through $S$. Since $P S$ has slope $q / p$, the first has exactly $t+1, t=[q / p]$, points of the lattice $\frac{1}{2} \mathbb{Z}^{3}$, of which one, $Q$, is on the boundary. ${ }^{5}$ Since the parallelogram intercepts the same length of each "vertical" it follows that all have either $t$ or $t+1$ points of $\frac{1}{2} \mathbb{Z}^{3}$.

Next, consider the $X$-GON $\mathcal{P}_{X}$, i.e., the undirected simple closed edge path consisting of the first and the last latitude, and the two edge paths obtained by joining the initial, respectively final, vertex of each latitude, to the initial, respectively final, vertex of the next latitude. Thus the two

\footnotetext{
${ }^{5}$ For the same reason, the $i$ th "vertical" has $[i q / p]-[(i-1) q / p]$ points.
} 
chains $\mathcal{C}$ which run on $\mathcal{P}_{X}$ from $X_{1}$ to $X_{q-1}$ are both almost maximal, and each of the $q-1$ points $X_{i}$ is either a vertex of $\mathcal{P}_{X}$, or is enclosed within it.

So, it only remains to check that $\mathcal{P}_{X}$ is convex iff $p$ divides $q-1$ or $q+1$. Convexity is equivalent to saying that the internal angle at each vertex $v$ of $\mathcal{P}_{X}$ is at most $180^{\circ}$. By central symmetry we need to consider only the initial vertices $v$ of the latitudes. At the initial vertices of the first and $p$ th latitudes the angle is clearly less than $180^{\circ}$. However, at an initial vertex $v$ of any other latitude the internal angle of $\mathcal{P}_{X}$ exceeds $180^{\circ}$ iff the preceding latitude has more vertices (i.e., iff, of the edges of $\mathcal{P}_{X}$ meeting in $v$, the one on the left has slope, $t+1$, one more than the one on the right). Thus, either all the parts of the $X$-partition have the same size $t$, or only the initial and final parts are of size $t$, and all others are of size $t+1$. So, either $q-1=p t$, or $q-1=2 t+(p-2)(t+1)$, i.e., $q+1=p(t+1)$.

Remark 7. We thus have $\frac{1}{4} \mathbb{Z}^{3}$-primitive subdivisions of many elementary tetrahedra, e.g., all those isomorphic to a $\mathbf{T}(p, q)$ with $p \leq 4$, and, using Lemma 3 , this covers all having $q \leq 11$. The case $(p, q)=(5,12)$ is the smallest which escapes Proposition 7, and now, not only is the $X$-gon $\mathcal{P}_{X}$ non-convex, its intersection with the maximal $Y$-chain - shown dotted in Figure $13 \mathrm{a}$ - is not a subcomplex. So, if $\mathcal{C}$ is on $\mathcal{P}_{X}$, the almost maximal $Y$-chain $\mathcal{D}$ cannot be arbitrary. But, in an amazingly large number of cases, including all with $q \leq 20$, the intersection $\mathcal{P}_{X} \cap \mathcal{P}_{Y}$ of the $X$-gon and the $Y$-gon is a subcomplex, e.g., as Figure $13 \mathrm{~b}$ shows, if $(p, q)=(7,19)$, then $\mathcal{P}_{X} \cap \mathcal{P}_{Y}$ consists of 6 edges and their 10 vertices. In all these cases, we obtain a $\frac{1}{4} \mathbb{Z}^{3}$-primitive subdivision by choosing the complementary $\mathcal{D}$ on $\mathcal{P}_{Y}$. However, $\mathcal{P}_{X} \cap \mathcal{P}_{Y}$ is not always a subcomplex: Figure 14 shows that for the case $(p, q)=(12,31), \mathcal{P}_{X}$ and $\mathcal{P}_{Y}$ have no common edge, but $\mathcal{P}_{X} \cap \mathcal{P}_{Y}$ contains, besides 22 vertices, 4 other isolated points. Now, for a pair $(\mathcal{C}, \mathcal{D})$ with $\mathcal{C} \subset \mathcal{P}_{X}$ and $\mathcal{D} \subset \mathcal{P}_{Y}, \mathcal{C} \cap \mathcal{D}$ is not a subcomplex (but, using a small detour in one of the 2 chains, one again gets a pair of almost maximal chains with intersection a subcomplex).

We do not need Proposition 7, or any of the constructions just mentioned, in the proof of Theorem 2. However they are of independent interest, and are what first convinced us about the truth of this theorem. In all these constructions, we stayed away from the barycenter $M$, thinking that this was needed to avoid bad intersections. However, somewhat surprisingly, "moving towards $M$ " is exactly what gave us a general construction of the required chain pairs. 
$(5,12)$

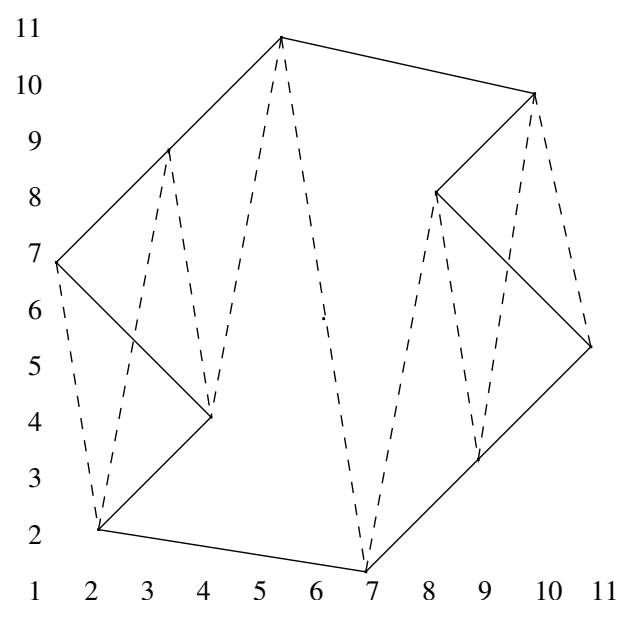

Figure 13a.

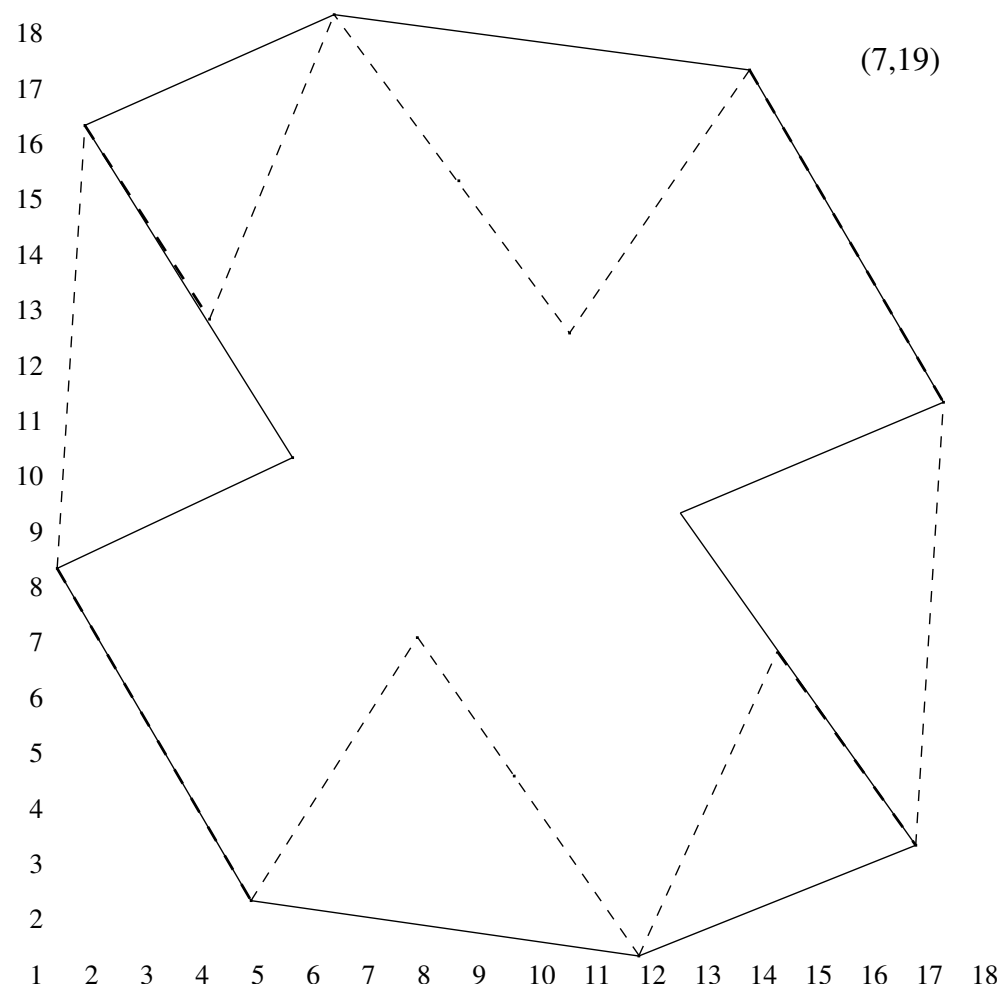

Figure 13b. 
8
7

$$
6
$$

5
4

$$
3
$$$$
2
$$$$
\text { , }
$$

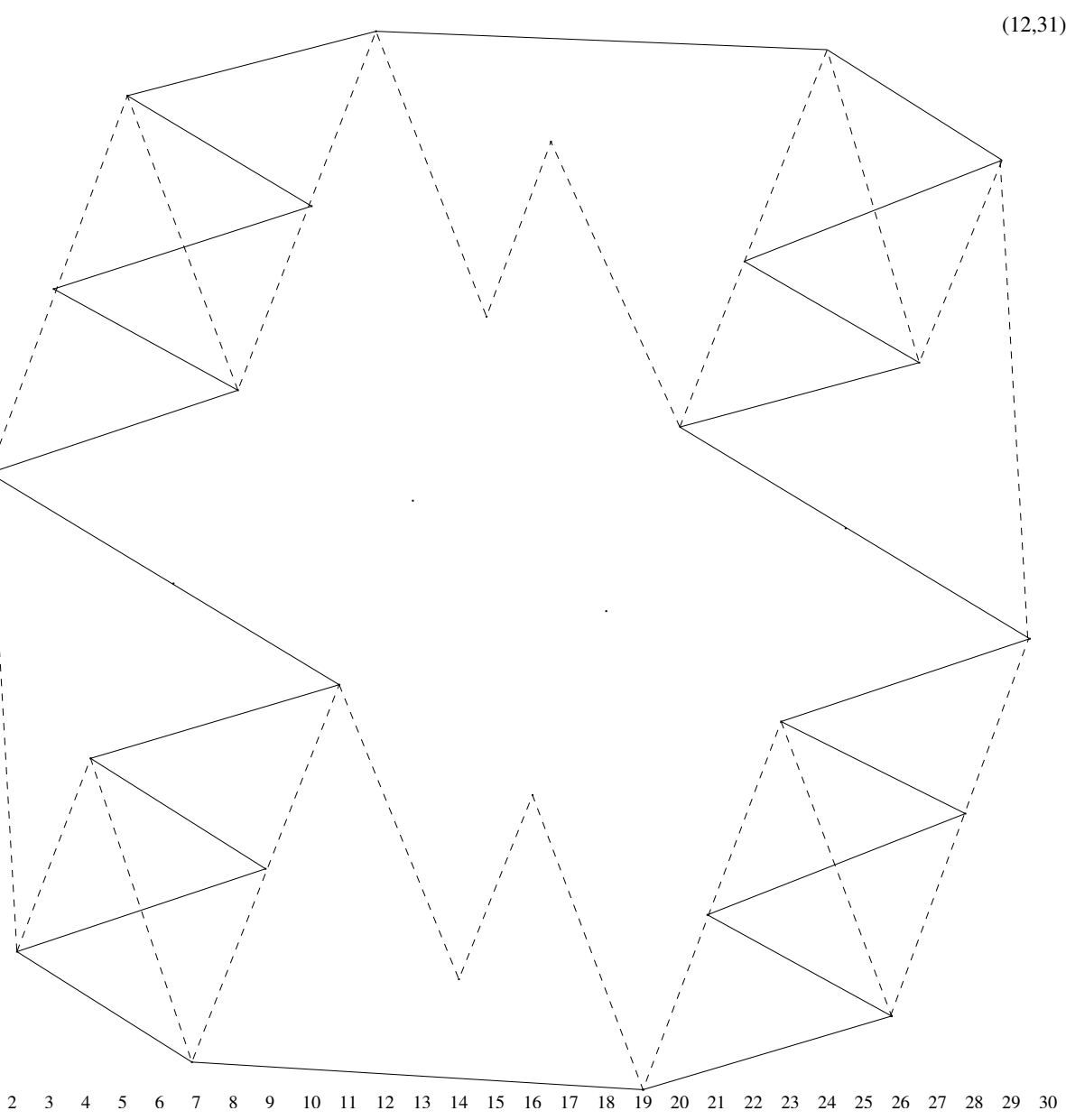

Figure 14.

QUADRANTS. Choosing $M$ as "origin" we draw - see Figure 12 - through $M$ two "axes" in the directions parallel to the latitudes and the longitudes. If $q$ is even, $M \in \frac{1}{2} \mathbb{Z}^{3}$, and each "axis" is itself a latitude or longitude; however, for $q$ odd, when $M \notin \frac{1}{2} \mathbb{Z}^{3}$, it may or may not be one. We adopt the usual terminology for the four parts of the parallelogram, e.g., the "first quadrant" is that which contains $R$. The idea now is to (essentially) confine $\mathcal{C}$ and $\mathcal{D}$ to distinct pairs of opposite quadrants: Since then $M \in \mathcal{C} \cap \mathcal{D}$, this idea works best only for $q$ even, so this is the case which we will treat first.

Proposition 8. For all $q$ even, there is a pair $(\mathcal{C}, \mathcal{D})$ of centrally symmetric almost maximal chains, with $\mathcal{C} \cap \mathcal{D}=\{M\}$.

So, by the argument as before, all $\mathbf{T}(p, q)$ with $q$ even must have a $\frac{1}{4} \mathbb{Z}^{3}$ primitive subdivision. We note that in the following we will argue from the squared representation of the central section. 
Proof. First, suppose that the slopes of the "axes" have opposite signs, say, the latitudes have positive slope and the longitudes negative (the opposite case is similar). This is the case $q-p<q / 2<s$, and is the one that was shown in Figure 12. Note that the open third quadrant (i.e., that containing $P$ ) intercepts a length bigger than $P X_{1}$ from all latitudes, starting with the first, and before that through $M$. So we can choose, on each of these intercepts, one point of the lattice $\mu\left(\mathbb{Z}^{3}\right)$. Using these chosen points, the barycenter $M$, and the symmetric points of the first quadrant (i.e., that containing $R$ ), we obtain a vertex subsequence of the maximal $X$-chain $X_{1} X_{2} \ldots X_{q-1}$, which determines an almost maximal $X$-chain $\mathcal{C}$, which but for $M$, is contained in the interior of the third and first quadrants. Likewise, because the longitudinal intercepts contained in the open second quadrant have length bigger than $S Y_{1}$, we can define a centrally symmetric almost maximal $Y$-chain $\mathcal{D}$, which, but for $M$, is contained in the interior of the second and fourth quadrants. So $\mathcal{C} \cap \mathcal{D}=\{M\}$.

Now suppose that both latitudes and longitudes have positive slope (the opposite case when both slopes are negative is similar), i.e., that both $q-p$ and $s$ are less than $q / 2$. As shown in Figure 15, now $X_{1}$ and $Y_{1}$ may both be in the third quadrant, or one of them may be instead in the neighbouring, i.e., fourth or second respectively, quadrant, but we assert that one cannot have $X_{1}$ in the fourth quadrant and $Y_{1}$ in the second quadrant. For this to happen, the slope of $M X_{1}$, i.e., $(q / 2-1) \div(q / 2-(q-p))$, must exceed the slope $s$ of a longitude, and the slope of $M Y_{1}$, i.e., $(q / 2-s) \div(q / 2-1)$, must be less than the slope $1 /(q-p)$ of a latitude. So $q / 2-1$ has to be bigger than both $s(q / 2-(q-p))$ and $(q / 2-s)(q-p)$. If $s<q-p$ the second number is bigger, if $s=q-p$ both are equal, and if $s>q-p$ the first number is bigger. In any case the bigger of the numbers exceeds the minimum $(q / 4)^{2}$ of $x(q / 2-x), 0 \leq x \leq q / 2$. So $q / 2-1>q^{2} / 16$, i.e., $(q-4)^{2}<0$, which is absurd.

If say $Y_{1}$ is in the second quadrant, then, noting that the longitudinal intercept contained in this quadrant have a length bigger than $P Y_{1}$, we choose as above an almost maximal and symmetric $\mathcal{D}$ through the interiors of the second and fourth quadrants, except for the one point $M$. Also, the latitudinal intercepts in the third quadrant being of length at least $P X_{1}$, there is an analogous $\mathcal{C}$ through the third and first quadrant. The case when $X_{1}$ is in the fourth and $Y_{1}$ in the third quadrant, is exactly similar, only the quadrant pairs of the chains are now switched. So, in all these cases, $\mathcal{C} \cap \mathcal{D}=\{M\}$. 


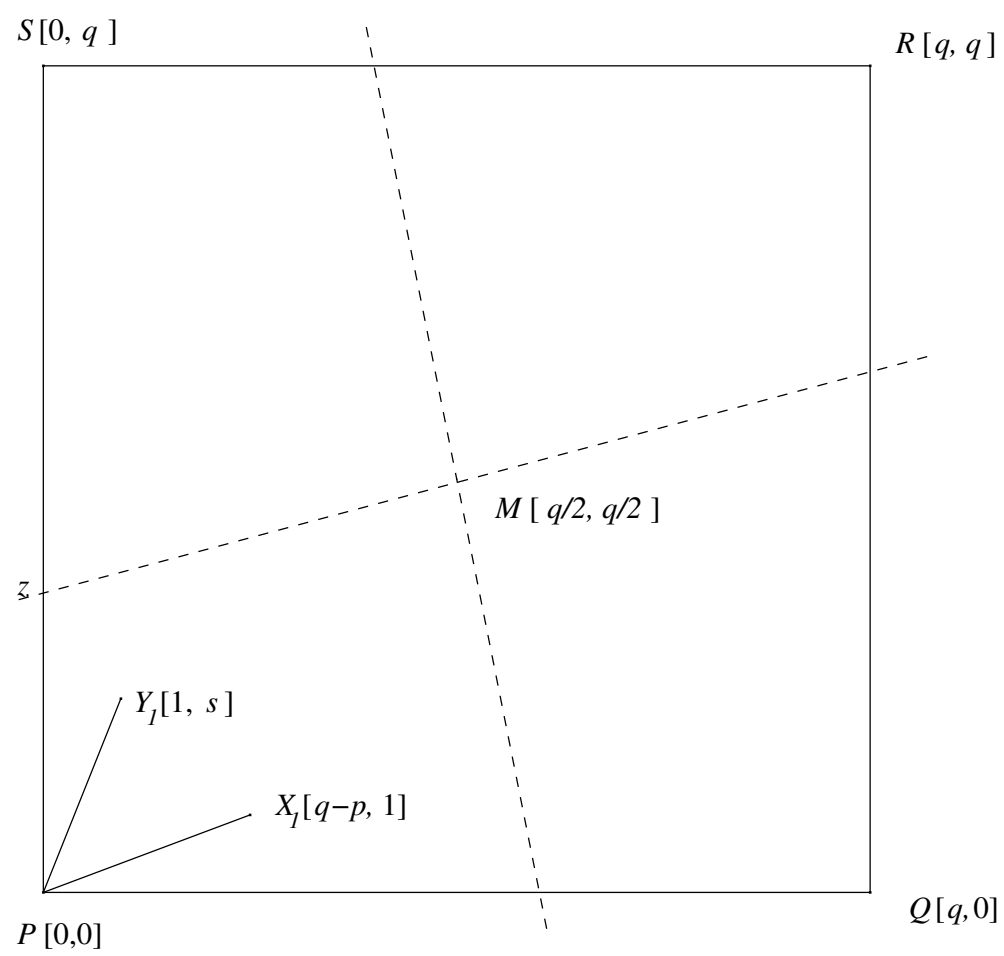

\section{Figure 15.}

There remains only the case of Figure 15, when of course $\mathcal{C}$ and $\mathcal{D}$ cannot be completely (but for $M$ ) in distinct pairs of opposite quadrants. Now choose one of the chains, say $\mathcal{D}$, just as before, so it runs through the third and first quadrants. Moreover, since the portion of the third quadrant lying above $P X_{1}$ produced also has longitudinal intercepts of length bigger than $P Y_{1}$, we can arrange that $\mathcal{D}$ is strictly above the first latitude, and so also strictly below the last latitude. For $\mathcal{C}$, we choose an "initial portion" on the first latitude till we are in the fourth quadrant. The "middle portion" of $\mathcal{C}$ runs between this and the symmetric "final portion", through the fourth and second quadrants, and is defined just as before using the fact that the latitudinal intercepts in the fourth quadrant have length exceeding $P X_{1}$. This gives again a symmetric almost maximal pair with $\mathcal{C} \cap \mathcal{D}=\{M\}$.

To finish the proof of Theorem 2 we will use, firstly, the fact that an analogous conclusion is valuable even if $q$ is odd.

Lemma 5. Any $\mathbf{T}(p, q)$ having a pair $(\mathcal{C}, \mathcal{D})$ of almost maximal chains with $\mathcal{C} \cap \mathcal{D}=\{M\}$ admits a $\frac{1}{4} \mathbb{Z}^{3}$-primitive subdivision. 
More generally, a similar construction shows that the same is true if $\mathcal{C}^{\prime} \cap \mathcal{D}^{\prime}$ is a subcomplex of $\mathcal{C}^{\prime}$ and $\mathcal{D}^{\prime}$, the subdivisions of $\mathcal{C}$ and $\mathcal{D}$ obtained by considering middle points of edges as new vertices.

Proof. For the remaining case $q$ odd, we will describe a modification of the previous construction, which only uses the fact that $M \in \frac{1}{4} \mathbb{Z}^{3}$. We begin by constructing the $\frac{1}{2} \mathbb{Z}^{3}$-elementary subdivisions $\mathbf{K}_{\mathcal{C}}(p, q)$ and $\mathbf{K}_{\mathcal{D}}(p, q)$, of the bottom and the top half, just as before, but take the following extra care in choosing the sub-triangulations $\mathbf{P}_{\mathcal{C}}(p, q)$ and $\mathbf{P}_{\mathcal{D}}(p, q)$, of the parallelogram $\mathbf{P}$, used in defining them.

Let $X \bar{X}$ and $Y \bar{Y}$ denote, respectively, the edges of $\mathcal{C}$ and $\mathcal{D}$ of which $M$ is the middle point. We choose a $\frac{1}{2} \mathbb{Z}^{3}$-elementary centrally symmetric polygonal region $\mathbf{R} \subseteq \mathbf{P}$, such that four of its vertices are $X, \bar{Y}, \bar{X}$, and $Y$. Note that this $\mathbf{R}$ can have other vertex pairs $\{E, \bar{E}\}$, and can be nonconvex, we only demand that if a segment having $M$ as its middle point has one end point in $\mathbf{R}$, then the entire segment be in $\mathbf{R}$. It is easily seen that such a polygon $\mathbf{R}$ can always be found. The extra care we now take is that the boundary of $\mathbf{R}$ be a subcomplex of both $\mathbf{P}_{\mathcal{C}}(p, q) \subset \mathbf{K}_{\mathcal{C}}(p, q)$ and $\mathbf{P}_{\mathcal{D}}(p, q) \subset \mathbf{K}_{\mathcal{D}}(p, q)$, and that $\mathbf{P}_{\mathcal{C}}(p, q)$ and $\mathbf{P}_{\mathcal{D}}(p, q)$ differ only on the interior of the polygonal region $\mathbf{R}$.

The triangulations $\mathbf{R}_{\mathcal{C}}(p, q)$ and $\mathbf{R}_{\mathcal{D}}(p, q)$ of this region have to be different, because $X \bar{X}$ is in the former, and $Y \bar{Y}$ in the latter. We nevertheless go ahead and subdivide $\mathbf{K}_{\mathcal{C}}(p, q)$ just as before to obtain a $\frac{1}{4} \mathbb{Z}^{3}$-primitive subdivision $\mathbf{K}_{\mathcal{C}}^{\prime}(p, q)$ of the bottom half. Recall that in this step the tetrahedra of the second kind are subdivided using Theorem 1, and the remaining tetrahedra, all primitive, are subdivided further by the method of Figure 9. The edge $X \bar{X}$ separates the interior of $\mathbf{R}$ into two parts, one contained in the left polygonal region of $\mathcal{C}$, and the other in the right polygonal region. Removing all simplices of $\mathbf{K}_{\mathcal{C}}^{\prime}(p, q)$ incident to the left part creates a TROUGH - see Figure 16 - whose bottom carries a triangulation similar to the left half of $\mathbf{R}_{\mathcal{C}}(p, q)$, and all walls of this trough are triangulated in the standard way. In particular, the triangulation of the wall of $X \bar{X}$ is a cone over $M$. So we can refill this trough by coning the remaining walls and bottom over $M$. We similarly create and refill a trough on the right side. This gives us a $\frac{1}{4} \mathbb{Z}^{3}$-primitive subdivision $\mathbf{K}_{\mathcal{C}}^{\prime \prime}(p, q)$ of the bottom half, which matches with the similarly modified $\frac{1}{4} \mathbb{Z}^{3}$-primitive subdivision $\mathbf{K}_{\mathcal{D}}^{\prime \prime}(p, q)$ of the top half, because, in both cases, the polygonal region $\mathbf{R}$ has now been retriangulated as the cone of its boundary over $M$. 


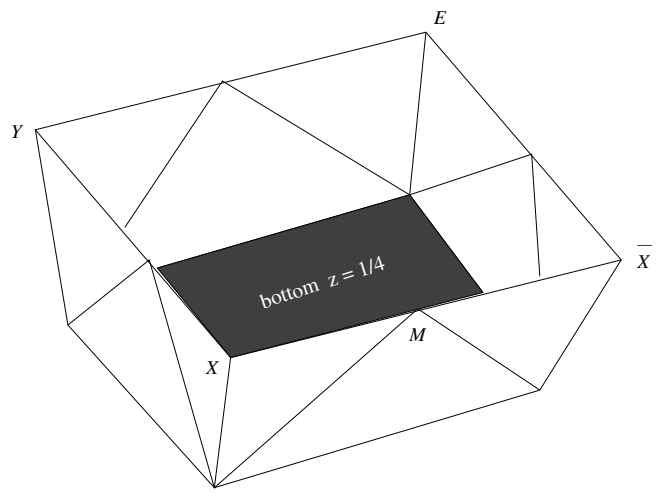

Figure 16.

Another point which we will use in the following is that we can employ chains slightly more general than almost maximal chains, namely, we can often SKIP a latitude (or longitude) provided it contains only two lattice points. For instance, if $q / p>2$, and $X_{j}$ is the last vertex of the previous latitude, then the first vertex of the next latitude is $X_{j+3}$, and using Lemma 2 we know that the join $X_{j} X_{j+3} *(A U$ or $U B)$ is isomorphic to $\frac{1}{2} \mathbf{T}(2,3)$, and so admits a $\frac{1}{4} \mathbb{Z}^{3}$-primitive subdivision.

Conclusion of proof of Theorem 2. For the remaining case $q$ odd, the argument of Proposition 8 applies verbatim, to yield a centrally symmetric almost maximal pair $(\mathcal{C}, \mathcal{D})$ with $\mathcal{C} \cap \mathcal{D}=\{M\}$, provided the numbers of latitudes and longitudes are both even (e.g., if $q-p$ and $s$ are even and less than $q / 2$ ). Using Lemma 5 we obtain the subdivision.

In all other cases, at least one of the "axes" has lattice points, the lattice points nearest to $M$ on such an "axis" being $\left\{X_{t}, X_{t+1}\right\}$ or $\left\{Y_{t}, Y_{t+1}\right\}$, where $t=(q-1) / 2$. We still need to make only a trifling change in the construction of $\mathcal{C}$ or $\mathcal{D}$, as described in the proof of Proposition 8, provided the slopes of the two "axes" have different signs. The trifling change being that we choose both $\left\{X_{t}, X_{t+1}\right\}$ or $\left\{Y_{t}, Y_{t+1}\right\}$ from this central latitude or longitude. This again gives a centrally symmetric almost maximal pair $(\mathcal{C}, \mathcal{D})$ with $\mathcal{C} \cap \mathcal{D}=\{M\}$.

This is however not permissible, for one of the two chains, when the slopes of the two "axes" have the same sign, say, positive as in Figure 15 (case negative is similar). Now, in the construction used to prove Proposition 8, one of the two chains, say $\mathcal{C}$, had either not started from the third quadrant, or else had been diverted from it, by means of an "initial portion" on the first latitude, into the neighbouring fourth quadrant, and then $\mathcal{C}$ had remained, till $M$, in this quadrant. This entails choosing $X_{t+1}$ before $X_{t}$, which is not allowed.

This difficulty is easily overcome if $\left\{X_{t}, X_{t+1}\right\}$ happen to be the only lattice points of the central latitude. Now, while defining $\mathcal{C}$, we choose the 
last vertex $X_{t-1}$ of the previous latitude, which is in the fourth quadrant, then skip both $\left\{X_{t}, X_{t+1}\right\}$, and move on to the symmetric first vertex $X_{t+2}$ of the next latitude, which is in the second quadrant. Once again we obtain a pair $(\mathcal{C}, \mathcal{D})$ of centrally symmetric chains with $\mathcal{C} \cap \mathcal{D}=\{M\}$, and, using the remark made above, one can still use these chains as before to make a $\frac{1}{4} \mathbb{Z}^{3}$-primitive subdivision. In case it is only the central longitude which has two lattice points, and one of the two chains needs to be diverted, we take care to divert $\mathcal{D}$, not $\mathcal{C}$.

If the number of lattice points on each "axis" is more than two, i.e., four or more, we give up central symmetry, and overcome the above difficulty by SHIFTING THE ORIGIN from $M$ to the lattice point $X_{t}$ (or $Y_{t}$ ) the new "quadrants" being now those which are determined by the latitude and longitude through $X_{t}$. Because there are at least 4 lattice points on the central latitude, the distance from $X_{t}$ to $Z$, the point where this latitude intersects $P S$, is bigger than $P X_{1}$, thus guaranteeing that all latitudinal intercepts contained in the (now smaller) second quadrant still have length bigger than $P X_{1}$. Thus $\mathcal{C}$ can be defined like before: An initial diversion on the first latitude into the fourth quadrant, then a lattice point from each latitude in this (now bigger) quadrant till the point $X_{t}$, after which we can continue like this in the third quadrant, thanks to the observation just made, till we meet the final latitude, along which we finally go to $X_{q-1}$. The other chain $\mathcal{D}$ is in the new third and first quadrants, and definable just as before, because the longitudinal intercepts contained in them, and between the first and final latitudes, have lengths bigger than $P Y_{1}$. This gives a (non-symmetric) pair of almost maximal chains $\mathcal{C}$ and $\mathcal{D}$, whose intersection is the subcomplex consisting of the single lattice point $X_{t}$. So once again a $\frac{1}{4} \mathbb{Z}^{3}$-primitive subdivision exists.

\section{References}

[Da] V.I. Danilov, Birational geometry of toric 3-folds, Math. USSR Izv., 21 (1983), 269-280, MR 84e:14008, Zbl 0536.14008.

[GKZ] I.M. Gelfand, M.M. Kapranov and A.V. Zelevinsky, Discriminants, Resultants and Multidimensional Determinants, Birkhaüser, Basel, 1994, MR 95e:14045, Zbl 0827.14036.

[Hu] J.F.P. Hudson, Piecewise Linear Topology, Benjamin, New York, 1969, MR 40 \#2094, Zbl 0189.54507.

[Ka] J.-M. Kantor, Triangulations of integral polytopes and Ehrhart polynommials, Beitr. Algebra Geom., 39 (1998), 205-218, MR 99b:52024, Zbl 0893.52008.

[Ka2] _ On the width of lattice-free simplices, Compos. Math., 118 (1999), 235241, MR 2001h:52011, Zbl 0936.52004

[KKMS] G. Kempf, F. Knudsen, D. Mumford and B. Saint Donat, Toroidal Embeddings I, Springer Lecture Notes, 339, 1973, MR 49 \#299, Zbl 0271.14017. 
[Re] J.E. Reeve, On the volume of lattice polyhedra, Proc. Lond. Math. Soc., 7 (1957), 378-395, MR 20 \#1954, Zbl 0080.26701.

[Rez] B. Reznick, Lattice point simplices, Discrete Math., 60 (1986), 219-242, MR 87i:52022, Zbl 0593.52008.

[Wh] G.K. White, Lattice tetrahedra, Can. J. Math., 16 (1964), 389-396, MR 28 \#5041, Zbl 0124.02901.

Received March 24, 2002 and revised October 23, 2002.

Institut de Mathematique De Jussieu

175, Rue DU Chevaleret

75013 PARIS

FRANCE

E-mail address: kantor@math.jussieu.fr

Department of Mathematics

PANJAB UNIVERSITY

ChANDigarh 160014

INDIA

E-mail address: sarkaria_2000@yahoo.com 